\title{
Taxonomic study on a collection of terrestrial mollusks from the region of Santa Maria, Rio Grande do Sul state, Brazil
}

\author{
Fernanda Santos Silva ${ }^{1,3}$; Luiz Ricardo L. Simone ${ }^{1,4}$ \& Rodrigo Brincalepe Salvador ${ }^{2}$ \\ 1 Universidade de São Paulo (USP), Museu de Zoologia (MZUSP). São Paulo, SP, Brasil. \\ ${ }^{2}$ Museum of New Zealand Te Papa Tongarewa. Wellington, New Zealand. \\ ORCID: http://orcid.org/0000-0002-4238-2276. E-mail: salvador.rodrigo.b@gmail.com \\ ${ }^{3}$ ORCID: http://orcid.org/0000-0002-2213-0135. E-mail: fernanda06@usp.br (corresponding author) \\ ${ }^{4}$ ORCID: http://orcid.org/0000-0002-1397-9823. E-mail: Irsimone@usp.br
}

\begin{abstract}
The malacological collection of the Universidade Federal de Santa Maria, curated by Dr. Carla B. Kotzian, has been recently donated to the Museu de Zoologia da Universidade de São Paulo (MZSP, Brazil). The collection is rich in well preserved specimens of terrestrial gastropods from central Rio Grande do Sul state, in southernmost Brazil. That region, centered in the municipality of Santa Maria, represents a transitional area between the Atlantic Rainforest and Pampas biomes and has been scarcely reported in the literature. Therefore, we present a taxonomical study of these specimens, complemented by historical material of the MZSP collection. Overall, we report 20 species, mostly belonging to the Stylommatophora, from which four represent new records for Rio Grande do Sul: Adelopoma brasiliense, Happia vitrina, Macrodontes gargantua, and Cyclodontina corderoi. The present report of C. corderoi is also the first from Brazil. Two introduced species were found in the studied material: Bradybaena similaris and Zonitoides sp.
\end{abstract}

Key-Words. Diplommatinidae; Gastropoda; Helicinidae; Pulmonata; Stylommatophora.

\begin{abstract}
Resumo. A coleção malacológica da Universidade Federal de Santa Matia, curada pela Dra. Carla B. Kotzian, foi recentemente doada ao Museu de Zoologia da Universidade de São Paulo (MZSP, Brasil). A coleção é rica em espécimes bem preservados de gastrópodes terrestres do estado do Rio Grande do Sul, no extremo sul do Brasil. Esta região, centrada no município de Santa Maria, representa uma área de transição entre os biomas Mata Atlântica e Pampa, e tem sido pouco relatada na literatura. Visando melhoria desse cenário, apresentamos um estudo taxonômico desses espécimes, complementado por material histórico da coleção MZSP. No total, relatamos 20 espécies, na sua maioria pertencentes a Stylommatophora, das quais quatro representam novos registros para o Rio Grande do Sul: Adelopoma brasiliense, Happia vitrina, Macrodontes gargantua e Cyclodontina corderoi. 0 presente relato de C. corderoi é também o primeiro do Brasil. Duas espécies introduzidas foram encontradas no material estudado: Bradybaena similaris e Zonitoides sp.
\end{abstract}

Palavras-Chave. Diplommatinidae; Gastropoda; Helicinidae; Pulmonata; Stylommatophora.

\section{INTRODUCTION}

Recently, Dr. Carla Bender Kotzian, who worked in the Universidade Federal de Santa Maria (UFSM, Brazil), transfered the collection guard of Recent mollusks to the Museu de Zoologia da Universidade de São Paulo (MZSP, Brazil). Among the material of this collection, our attention was drawn to the specimens of terrestrial gastropods from the central region of Rio Grande do Sul state (centered in the municipality of Santa Maria), which proved to be rather abundant and in a good preservation state.
Therefore, with the intention of bringing new information on the terrestrial gastropod fauna of a scarcely sampled region, we present herein the species from the Santa Maria region found in the former UFSM collection, complemented by further historical material deposited in the collection of the MZSP. All the species are figured below, with notes on their distribution, diagnosis and taxonomy, when applicable. Overall, we report 20 species, mostly belonging to Stylommatophora, from which three represent new distribution records. 


\section{MATERIAL AND METHODS}

All the studied specimens stem from several municipalities around Santa Maria (Fig. 1) and are now deposited in the malacological collection of the MZSP (São Paulo, Brazil). The full list of analyzed material, with lot numbers and collection data, can be found under each species entry below. Identification of the species was based in specialized literature: original descriptions, further taxonomic literature, the catalogue by Simone (2006), and comparative material from the MZSP collection. Species are presented below in systematic order, following Bouchet et al. (2017). Coordinates given on the text are for the city center of each municipality, as the collection events here typically lack this data. Measurements were made either with a digital caliper (for larger specimens) or with the aid of the Zeiss Axiovision SE64 Rel 4.8 imaging software. The following abbreviations are used herein: $L=$ shell length; $D=$ shell greatest width (perpendicular to $\mathrm{L}$ ); RS = Rio Grande do Sul state; UFSM = former Universidade Federal de Santa Maria collection; $c o l=$ collector(s); sh = shell(s); spm = specimen(s).

\section{SYSTEMATICS \\ Neritimorpha \\ Superfamily Helicinoidea \\ Family Helicinidae \\ Genus Helicina Lamarck, 1799 \\ Helicina densestriata Wagner, 1910}

(Fig. 2A-E)

Helicina densestriata Wagner, 1910: 272, pl. 53, figs. 11-13:

Shade, 1965: 213; Salgado \& Coelho, 2003: 151.

Helicina (Oxyrhombus) densestriata: Haas, 1959: 365.

Oxyrhombus densestriatus: Simone, 2006: 41, fig. 34; Agudo-Padrón, 2008: 51.

Type locality: Brazil, Rio Grande do Sul state, Serra do Mar.

Distribution: Brazil (Santa Catarina and Rio Grande do Sul states) and Paraguay (Simone, 2006).

Material studied: Brazil, Rio Grande do Sul: Agudo $\left(29^{\circ} 38^{\prime} \mathrm{S}, 53^{\circ} 15^{\prime} \mathrm{W}\right)$ : 6 sh, 21.x.1988, UFSM, L. Indrusiak

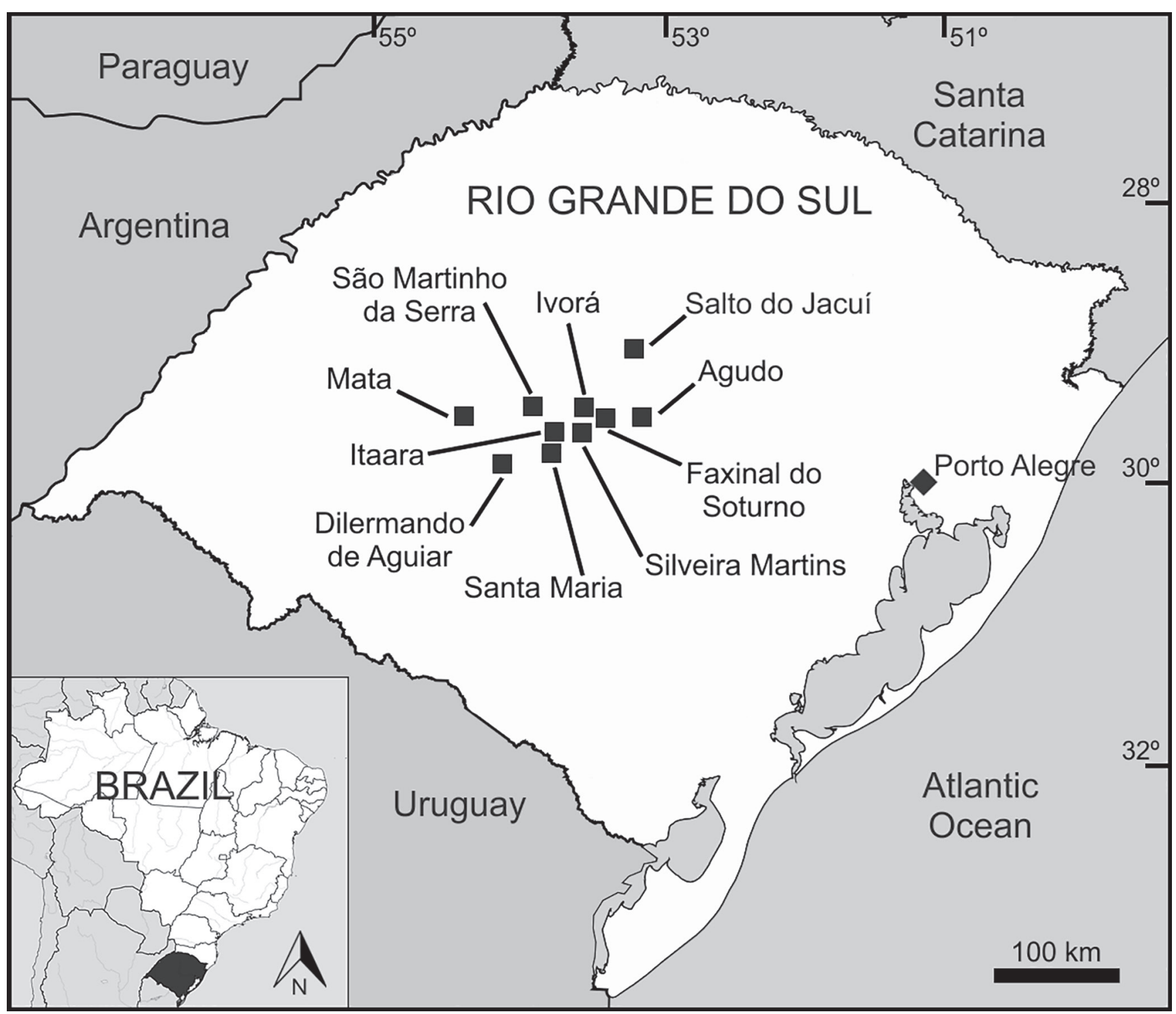

Figure 1. Map of Rio Grande do Sul state, showing the municipalities where the present specimens were collected. 
col. (MZSP 137933), 4 sh, 01.x.1988, UFSM, L. Indrusiak

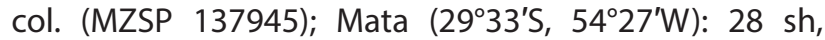
01.vi.1982, UFSM, M.L. Bier col. (MZSP 137938); Santa Maria $\left(29^{\circ} 41^{\prime} S, 53^{\circ} 48^{\prime} \mathrm{W}\right)$ : 7 sh, 21.vi.1979, A. Assunção col. (MZSP 43120); 2 sh, ix.1980, L. Indrusiak col. (MZSP 43339); 2 spm, ix.1980, L. Indrusiak col. (MZSP 44147);

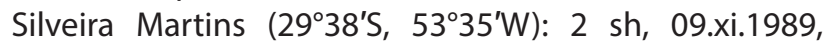
UFSM, L. Indrusiak col. (MZSP 137942).

Remarks: This species can be identified by its relatively small size and strong spiral cords on the teleoconch (on adapical area of the whorl, above the keel). As common for other congeners (e.g., Salvador et al., 2014), specimens of $H$. densestriata can vary from a rounded profile (Fig. 2D) to a strongly marked keel (Fig. 2A); furthermore, the spiral sculpture, although typically well marked, can be fainter in some specimens (Fig. 2B).

\section{Caenogastropoda \\ Superfamily Cyclophoroidea \\ Family Diplommatinidae \\ Genus Adelopoma Doering, 1885 \\ Adelopoma brasiliense Morretes, 1953} (Fig. 2F-G)

Adelopoma brasiliense Morretes, 1953: 331; Salgado \& Coelho, 2003: 153; Simone, 2006: 46, fig. 56; AgudoPadrón, 2014: 10; Martins \& Simone, 2014: 765; Salvador et al., 2018a: 106, figs. 5A-C.

Type locality: Brazil, São Paulo state, Iguape municipality.

Distribution: Brazil (São Paulo state and possibly Alagoas state) (Simone, 2006; Salvador et al., 2018a).

Material studied: Brazil, Rio Grande do Sul: Santa Maria (2941'S, 5348'W): 1 sh, ix.1990, UFSM, L. Indrusiak col. (MZSP 132596); 3 sh, ii.1990, UFSM, L. Indrusiak col.

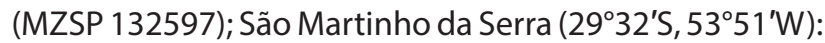
2 sh, v.1990, UFSM, L. Indrusiak col. (MZSP 132598); 4 sh, iv.1991, UFSM, L. Indrusiak col. (MZSP 132599);

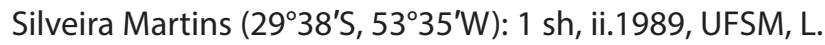
Indrusiak col. (MZSP 132595).

Remarks: The species can be distinguished from local congeners by its conical shell profile, with an acuminated and somewhat truncated apex, and the widely spaced axial ribs. The present record extends the species distribution to southern Brazil.

\section{Stylommatophora \\ Superfamily Achatinoidea \\ Family Achatinidae \\ Genus Lamellaxis Strebel \& Pfeffer, 1882 Lamellaxis gracile (Hutton, 1834)}

(Fig. 2H-I)

Bulimus gracilis Hutton, 1834: 93; Martens, 1973: 183.
Opeas gracile: Baker, 1913: 644; Vernhout, 1914: 18; Pilsbry, 1930a: 342; Haas, 1935: 111, 1939: 268; Morretes, 1949: 132; Jaeckel, 1952: 6.

Opeas (Synopeas) gracile: Baker, 1927: 7.

Opeas (Opeas) gracile: Haas, 1953: 205.

Lamelaxis gracilis: Richards \& Hummelinck, 1940: 9; Pilsbry, 1946: 177, figs. 85f-g; Morretes, 1953:62; Tillier, 1980:60, pl. 3, fig. 14; Cuezzo \& Drahg, 1995: 191; Oliveira \& Almeida, 1999: 23; Bruschi-Figueiró \& VeitenheimerMendes, 2002: 32; Salgado \& Coelho, 2003: 155; Silva \& Castro, 2003: 67; Simone, 2006: 184, fig. 669; AgudoPadrón, 2008: 157; Rumi et al., 2010: 2988.

Lamelaxis (Allopeas) gracilis: Baker, 1945: 88; Figueiras, 1963: 88; Shade, 1965: 215; Fernandez \& Castellanos, 1973: 271, pl. 4, fig. 3; Quintana, 1982: 81.

Lamelaxis gracile: Delannoye et al., 2015: 258, pl. 51.

Allopeas gracilis: Thompson, 2011: 159.

Allopeas gracile: Capinera, 2017: 116.

Type locality: India, Mirzapur.

Distribution: W. Indies, Central America, tropical and subtropical South America (Simone, 2006).

Material studied: Brazil, Rio Grande do Sul: Santa Maria (2941'S, 5348'W): 11 sh, 1987, UFSM, C.M. Ferreira col. (MZSP 132622); 1 sh, ix.1980, UFSM, L. Indrusiak col. (MZSP 132623).

Remarks: This species has been introduced in several tropical and subtropical localities worldwide (Capinera, 2017), but it likely originates from South America, despite its type locality (Delannoye et al., 2015). It is known throughout Brazil, including Rio Grande do Sul state (Simone, 2006).

\section{Genus Leptinaria Beck, 1837 Leptinaria lamellata (Potiez \& Michaud, 1838)} (Fig. 2J-K)

Helix unilamellata d'Orbigny 1835: 9 [nomen nudum]. Achatina lamellate Potiez \& Michaud, 1838: 128, pl. 11, figs. 7-8; Reeve, 1849a: pl. 18, fig. 97.

Leptinaria (Leptinaria) lamellata: Haas, 1953, 205.

Leptinaria lamellata: Pilsbry, 1906: 288; Baker, 1913: 645, 1927: 22; Haas, 1939: 268; Richards \& Hummelinck, 1940: 10; Jaeckel, 1952: 6; Götting, 1978: 105, fig. 7; Tillier, 1980: 62, pl. 3, fig. 12; Ramírez et al., 2003: 276; Salgado \& Coelho, 2003: 155; Simone, 2006: 186, fig. 678; Delannoye et al., 2015: 264, pl. 54; Salvador et al., 2018a: 116, figs. 10E-F; Salvador, 2019a: 91.

Leptinaria lamellata lamellata: Thompson, 2011: 164. Leptinaria (Leptinaria) unilamellata: Arias, 1953: 250, fig. 4. Leptinaria unilamellata: Baker, 1945: 91; Pilsbry, 1906: 288; Altena, 1960: 50; Dutra, 1988: 581; Araujo \& Keller, 1993: 502; Oliveira \& Almeida, 1999: 23; Silva \& Castro, 2003: 67; Simone, 2006: 186, fig. 684; Agudo-Padrón, 2008: 157; Massemin et al., 2009: 415, pl. 9, fig. D; Breure \& Araujo, 2017: 90, fig. 34B. 


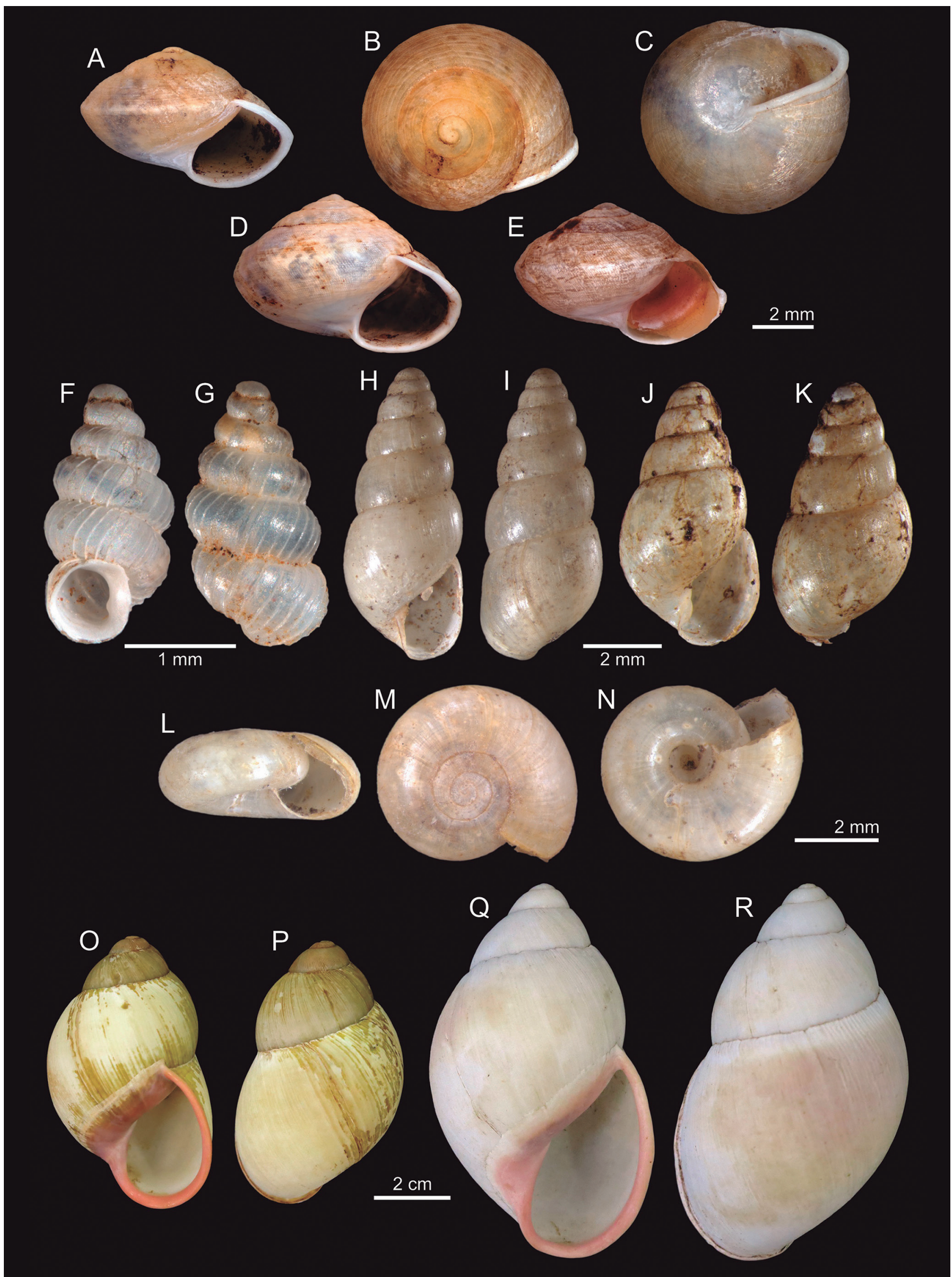

Figure 2. $(A-R)=$ Shells from Santa Maria region, Rio Grande do Sul state. $(A-E)=$ Helicina densestriata, $(A-C)=M Z S P 137933(L=4.6 \mathrm{~mm}, D=7.1 \mathrm{~mm})$. (D) $=$ MZSP $43339(\mathrm{~L}=5.2 \mathrm{~mm}, \mathrm{D}=7.6 \mathrm{~mm})$. $(\mathrm{E})=\mathrm{MZSP} 44147(\mathrm{~L}=4.6 \mathrm{~mm}, \mathrm{D}=6.9 \mathrm{~mm}) .(\mathrm{F}-\mathrm{G})=$ Adelopoma brasiliense MZSP $132597(\mathrm{~L}=2.3 \mathrm{~mm}) .(\mathrm{H}-\mathrm{I})=$ Lamellaxis gracile MZSP $132622(\mathrm{~L}=7.2 \mathrm{~mm})$. $(\mathrm{J}-\mathrm{K})=$ Leptinaria lamellata MZSP $132621(\mathrm{~L}=6.8 \mathrm{~mm}) .(\mathrm{L}-\mathrm{N})=$ Happia vitrina MZSP $132658(\mathrm{~L}=2.3 \mathrm{~mm}, \mathrm{D}=4.8 \mathrm{~mm}) .(0-\mathrm{P})=$ Megalobulimus abbreviatus MZSP 132550 ( $(=72 \mathrm{~mm}) .(\mathrm{Q}-\mathrm{R})=$ Megalobulimus haemastomus MZSP $132553(\mathrm{~L}=101 \mathrm{~mm})$. 
Type locality: Undefined, but Delannoye et al. (2015) indicated Santa Cruz department, in Bolivia, as a possibility.

Distribution: From Nicaragua to Brazil (Simone, 2006; Delannoye et al., 2015).

Material studied: Brazil, Rio Grande do Sul: Santa Maria (2941'S, 534' W): 3 sh, ix.1993, UFSM, L. Indrusiak col. (MZSP 132621).

Remarks: This species is very widespread in South America, including southern Brazil (Simone, 2006). It has been introduced to some Caribbean Islands and French Polynesia (Massemin et al., 2009).

\section{Superfamily Scolodontoidea Family Scolodontidae \\ Genus Happia Bourguignat, 1889 \\ Happia vitrina (Wagner, 1827)}

(Fig. 2L-N)

Helix vitrina Wagner in Spix, 1827: 25, pl. 17, fig. 6.

Solarium imperforatum Spix, 1827: 25, pl. 17, fig. 6.

Streptaxis (Happia) tumescens Suter, 1900: 330, pl. 3, fig. 4-4b.

Happia vitrina: Gude, 1902: 234; Thiele, 1927: 318; Haas, 1935: 111; Morretes, 1949: 138; Salgado \& Coelho, 2003: 169; Agudo-Padrón, 2008: 165; Birckolz et al., 2016: table 1; Salvador et al., 2018a: 116, figs. 11A-C; Salvador, 2019a: 93.

Scolodonta (Happia) vitrina: Kobelt, 1906: 49, pl. 48, figs. 13-14.

Happiavitrina var. mülleri: Thiele, 1927: 318.

Happia (Happia) vitrina: Haas, 1953, 205; Zilch, 1960: 545, fig. 1904.

Streptaxis tumescens: Marshall, 1996: 42; Simone, 2006: 194, fig. 722.

Happia vitrina muelleri: Agudo-Padrón, 2008: 165.

Type locality: Brazil, "southern provinces" (Wagner in Spix, 1827).

Distribution: Brazil, from Alagoas state to Santa Catarina state (Salvador et al., 2018a).

Material studied: Brazil, Rio Grande do Sul: Santa Maria (2941'S, 5348'W): 1 spm, ix.1980, L. Indrusiak col. (MZSP 44145); 1 sh, ii.1992, UFSM, L. Indrusiak col. (MZSP

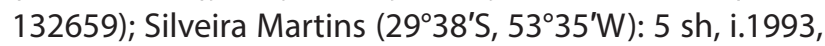
UFSM, L. Indrusiak col. (MZSP 132658).

Remarks: Even though shell characters are of limited use for diagnosis within the genus Happia, the present specimen confer with $H$. vitrina, a widespread species in Brazil, due to its depressed spire, relatively shallow umbilicus and abapically bent aperture. The present record extends the species distribution a little further to the south.

\section{Superfamily Rhytidoidea \\ Family Strophocheilidae \\ Genus Megalobulimus Miller, 187 \\ Megalobulimus abbreviatus (Bequaert, 1948)}

(Fig. 20-P)

Stropocheilus granulosus abbreviates Bequaert, 1948: 164, pl. 27, fig. 4.

Stropocheilus (Megalobulimus) granulosus abbreviatus: Sawaya \& Petersen, 1962: 37.

Megalobulimus abbreviatus: Bruschi-Figueiró \& Veitenheimer-Mendes, 2002: 33; Salgado \& Coelho, 2003: 157; Achaval et al., 2005: 74; Horn et al., 2005: 459; Agudo-Padrón \& Lenhard, 2011: 167; Borda \& Ramírez, 2013: 681; Agudo-Padrón, 2015: 4.

Type locality: Brazil.

Distribution: Brazil: Mato Grosso, São Paulo and Rio Grande do Sul states (Simone, 2006).

Material studied: Brazil, Rio Grande do Sul: Salto do Jacuí $\left(29^{\circ} 05^{\prime} \mathrm{S}, 5^{\circ} 12^{\prime} \mathrm{W}\right)$ : 6 sh, ii.1989, UFSM, G. Deprá

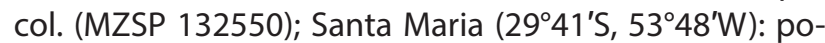
tentially lost; 1 sh, 1990, L. Indrusiak col. (MZSP 48216); 1 sh, v. 82, L. Indrusiak col. (MZSP 48241) 5 sh, 01.xii.1976, L. Indrusiak col. (MZSP 48244); Silveira Martins (29³8'S, $53^{\circ} 35^{\prime} \mathrm{W}$ ): 1 sh, ii.1989, UFSM, L. Indrusiak col. (MZSP 132569).

Remarks: Megalobulimus abbreviatus was originally described as a subspecies. However, Leme (1973) elevated all subspecies in Megalobuliminae to species level in an unpublished thesis that largely followed the faunal lists of Morretes (1949, 1953) (Fontenelle et al., 2019). Leme (1973) did not provide a clear rationale for his decision, but was nevertheless followed by later authors (e.g., Salgado \& Coelho, 2003; Simone, 2006). As a result, most Brazilian Megalobulimus spp. are in dire need of reassessment.

\section{Megalobulimus haemastomus (Scopoli, 1786)}

(Fig. 2Q-R)

Bulimus haemastomus Scopoli, 1786: 67, pl. 25, figs. B1-2; Lamarck, 1822: 117; Swainson, 1840: 180; Gray, 1847: 174; Troschel, 1849: 227.

Bulimus hämastomus: Küster, 1850: 5, pl. 1, figs. 3-4.

Strophocheilus oblongus haemastomus: Bequaert, 1948: 60, pl. 6, fig. 2, pl. 21, fig. 4, pl. 24, fig. 5; Barattini \& Ledón, 1949: 2; Buckup \& Buckup, 1957: 34; Klappenbach, 1967: 42.

Psiloicus haemastomus: Morretes, 1952: 113.

Strophocheilus (Megalobulimus) oblongus haemastomus: Figueiras, 1963: 83; Parodiz, 1957a: 132; Sawaya \& Petersen, 1962: 37; Figueiras, 1963: 83; Fernandez \& Castellanos, 1973: 276.

Megalobulimus oblongus haemastomus: Quintana, 1982: 84; Miquel \& Aguirre, 2011: 112, fig. 12. 
Megalobulimus haemastomus: Salgado \& Coelho, 2003: 158; Simone, 2006: 211. Agudo-Padrón, 2008: 161, 2014: 16, 2015: 4; Fontenelle et al., 2014: 34; Salvador, 2019a: 92.

Type locality: Undefined.

Distribution: Brazil (central to south), Paraguay, Uruguay, and Argentina (Simone, 2006).

Material studied: Brazil, Rio Grande do Sul: Agudo (293's, 5315'W): 10 sh, vi.1988, UFSM, Equipe Piscicultura col. (MZSP 132553); 2 sh, v.1987, UFSM, H. Schumacher col. (MZSP 132566); Faxinal do Soturno

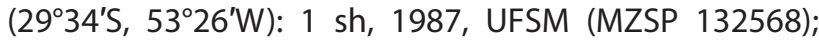

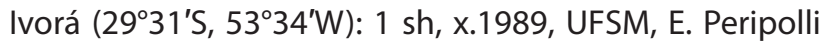
col. (MZSP 132565).

\section{Superfamily Succineoidea \\ Family Succineidae \\ Genus Succinea Draparnaud, 1801 \\ Succinea meridionalis d'Orbigny, 1837}

(Fig. 3A-B)

Succinea oblonga: d'Orbigny, 1837: 231 [non Draparnaud, 1835].

Succinea meridionalis d'Orbigny, 1837: 711; Fernandez \& Castellanos, 1973: 269; Doering, 1874: 52; Strobel, 1874:29; Pilsbry, 1911:520; Pfeiffer, 1876:47; Morretes, 1949: 130; Parodiz, 1957a: 129; Figueiras, 1963: 84; Shade, 1965: 214; Lanzieri, 1966: 170; Fernandez \& Castellanos, 1973: 269, pl. 1 fig. 3; Quintana, 1982: 75; Bruschi-Figueiró \& Veitenheimer-Mendes, 2002: 33; Salgado \& Coelho, 2003: 153; Scarabino, 2004: 269; Simone, 2006: 236, fig. 906; Agudo-Padrón, 2008: 156, 2014: 13; Santos et al., 2010: 512; Miquel \& Aguirre, 2011: 106, fig. 3; Miquel et al., 2015: 90.

Succinea (Hydrophyga?) meridionalis: Haas, 1953: 205.

Type locality: South America ("I'Amérique méridionale"; d'Orbigny, 1837: 231).

Distribution: From Rio de Janeiro state, Brazil, to Patagonia (Simone, 2006).

Material studied: Brazil, Rio Grande do Sul: Santa Maria (2941'S, 5348'W): 1 sh, 1 spm, 24.vii.1994, T. Breyer col. (MZSP 57504).

\section{Superfamily Orthalicoidea Family Bulimulidae \\ Genus Bulimulus Leach, 1814 \\ Bulimulus angustus Weyrauch, 1966}

(Fig. 3C-D)

Bulimulus (Bulimulus) versicalis angustus Weyrauch, 1966: 45; Breure, 1979: 61; Bruschi-Figueiró \& Veitenheimer-Mendes, 2002: 32.
Bulimulus versicalis: Richardson, 1995: 88.

Bulimulus angustus: Miquel, 1991: 94; Salgado \& Coelho, 2003: 160; Simone, 2006: 117, fig. 354; Agudo-Padrón, 2008: 158, 2014: 15; Agudo-Padrón \& Lenhard, 2011: 167; Birckolz et al., 2016: table 1.

Type locality: Brazil, Rio Grande do Sul state, São Leopoldo municipality.

Distribution: Brazil: Santa Catarina and Rio Grande do Sul states (Birckolz et al., 2016).

Material studied: Brazil, Rio Grande do Sul: Santa Maria (2941'S, 534'W): 1 spm, ix.1980, L. Indrusiak col. (MZSP 44144); 18sh, iv.1984, UFSM, L. Indrusiak col. (MZSP 132650); 2 sh, iii.1996, UFSM, L. Indrusiak col. (MZSP 132653).

\section{Bulimulus marcidus (Pfeiffer, 1853)}

(Fig. 3E-F)

Bulimus marcidus Pfeiffer, 1853: 435, 1854: 67; Pfeiffer, 1854 in Küster \& Pfeiffer, 1840-1865: 188, pl. 49 figs. 11-12; Hupé, 1857: 54.

Bulimulus marcidus: Pilsbry, 1901-1902: 146, pl. 25, figs. 23-24; Morretes, 1949: 147; Breure. 1979: 63; Richardson, 1995: 79; Salgado \& Coelho, 2003: 160; Simone, 2006: 118, fig. 364; Breure \& Ablett, 2014: 117, figs. 63I-J, L36v, 2015: 64.

Type locality: Brazil.

Distribution: Brazil (Simone, 2006).

Material studied: Brazil, Rio Grande do Sul: Santa Maria (29² $41^{\prime}$ S, $\left.53^{\circ} 48^{\prime} \mathrm{W}\right)$ : 7 sh, ix.1980, L. Indrusiak col. (MZSP 43336).

Remarks: This species can be identified by its medium size, convex whorls, and widely spaced prosocline axial ribs. The precise distribution of $B$. marcidus in Brazil is uncertain.

\section{Genus Drymaeus Albers, 1850 Drymaeus muelleggeri Jaeckel, 1927} (Fig. 3G-H)

Drymaeus muelleggeri Jaeckel, 1927: 136; Breure, 1979: 111; Richardson, 1995: 152; Salgado \& Coelho, 2003: 162; Simone, 2006: 140, fig. 463; Agudo-Padrón, 2014: 15.

Type locality: Brazil, Rio Grande do Sul state, Santa Rosa municipality.

Distribution: Brazil: Santa Catarina and Rio Grande do Sul states (Simone, 2006; Agudo-Padrón, 2014).

Material studied: Brazil, Rio Grande do Sul: Dilermando

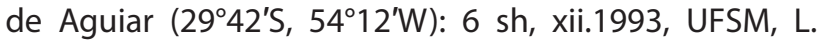




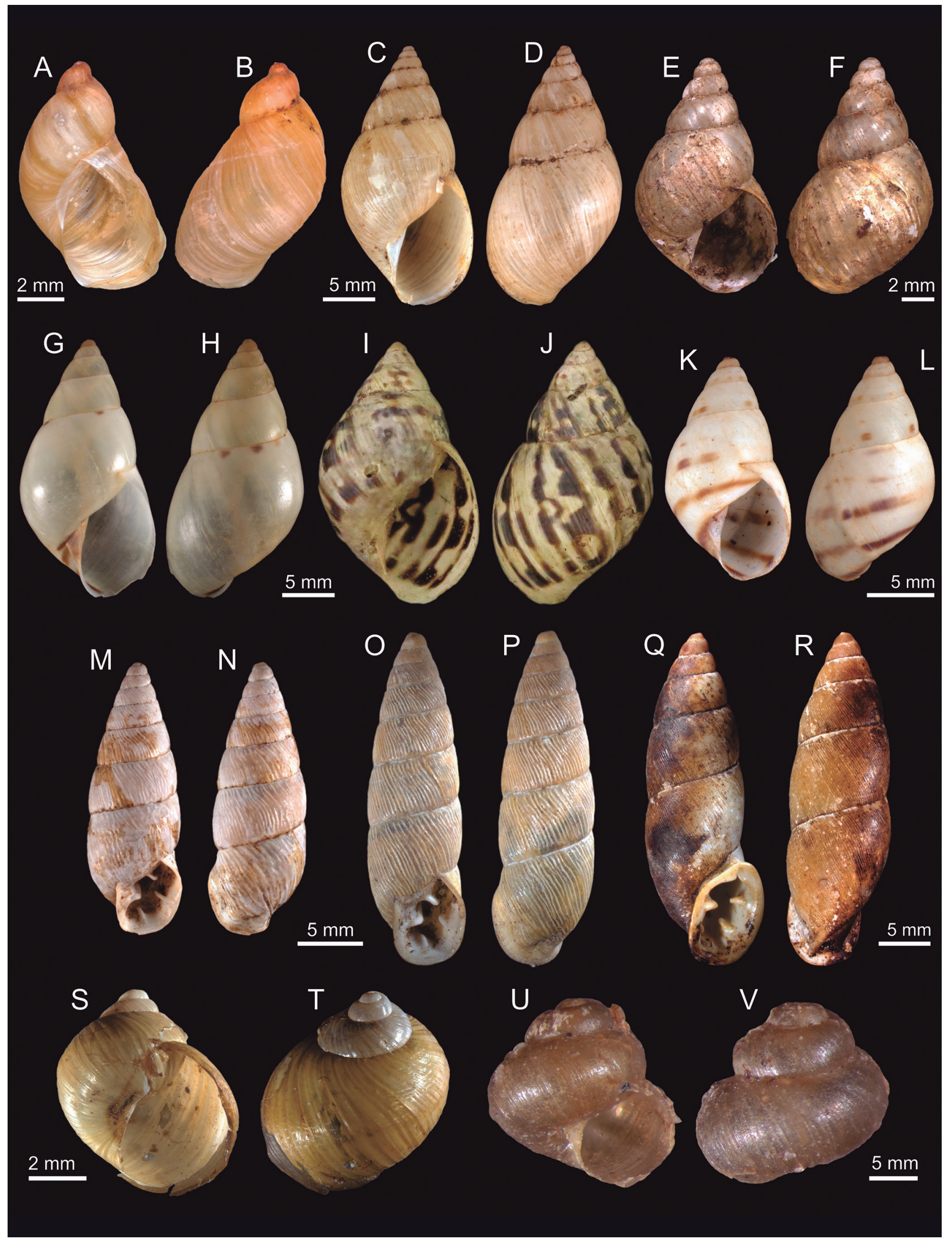

Figure 3. $(A-V)=$ Shells from Santa Maria region, Rio Grande do Sul state. $(A-B)=$ Succinea meridionalis MZSP $57504(L=9.7$ mm). (C-D) = Bulimulus angustus MZSP $132650(\mathrm{~L}=25.3 \mathrm{~mm})$. $(\mathrm{E}-\mathrm{F})=$ Bulimulus marcidus MZSP $43336(\mathrm{~L}=14.6 \mathrm{~mm}) .(\mathrm{G}-\mathrm{H})=$ Drymaeus muelleggeri MZSP $132577(\mathrm{~L}=24.8 \mathrm{~mm}) .(\mathrm{I}-\mathrm{J})=$ Drymaeus papyraceus MZSP $132558(\mathrm{~L}=25.0 \mathrm{~mm})$. $(\mathrm{K}-\mathrm{L})=$ Drymaeus poecilus MZSP $132588(\mathrm{~L}=16.9 \mathrm{~mm}) .(\mathrm{M}-\mathrm{N})=$ Cyclodontina corderoi MZSP $132605(\mathrm{~L}=21.1 \mathrm{~mm})$. $(0-P)=$ Cyclodontina corderoi MZSP $132609(\mathrm{~L}=25.7 \mathrm{~mm}) .(\mathrm{Q}-\mathrm{R})=$ Macrodontes gargantua MZSP $43332(\mathrm{~L}=32.7 \mathrm{~mm}) .(\mathrm{S}-\mathrm{T})=$ Simpulopsis sulculosa MZSP 132663 $(\mathrm{L}=7.3 \mathrm{~mm}, \mathrm{D}=6.1 \mathrm{~mm})$. $(\mathrm{U}-\mathrm{V})=$ Pupisoma dioscoricola MZSP $132671(\mathrm{~L}=2.0 \mathrm{~mm}, \mathrm{D}=2.1 \mathrm{~mm})$. 
Indrusiak col. (MZSP 132577); Mata (29 $\left.33^{\prime} \mathrm{S}, 5^{\circ} 27^{\prime} \mathrm{W}\right)$ : 1 sh, ii.1982, H.O. Lippold col. (MZSP 43122); Santa Maria $\left(29^{\circ} 41^{\prime} \mathrm{S}, 53^{\circ} 48^{\prime} \mathrm{W}\right)$ : 3 sh, (MZSP 43105).

\section{Drymaeus papyraceus (Mawe, 1823)}

(Fig. 3I-J)

Helix papyracea Mawe, 1823: 168, fig. 7; Breure, 1979: 112. Bulimus litus Férussac, 1819: 89, pl. 139, figs. 6, 7.

Bulimus lita: d'Orbigny, 1838: 268.

Bulimus papyraceus: Gray, 1825: 414; Beck, 1837: 65; Hupé, 1857: 52; Albers, 1860: 216; Heynemann, 1868: 110; Martens, 1868: 179; Hidalgo, 1869: 108, 1870: 57; Pfeiffer, 1848: 102; Reeve, 1849b: pl. 39, fig. 236; lhering, 1884: 271.

Bulimulus papyraceus: Ancey, 1897: 5.

Bulimulus (Mormus) papyraceus: Clessin, 1888: 166.

Drymaeus (Drymaeus) papyraceus: Breure \& Eskens, 1981: 32, figs. 100-101; Breure \& Araujo, 2017: 78, fig. 30D.

Drymaeus (Leiostracus) papyraceus: Haas, 1935: 110.

Drymaeus papyraceus: Pilsbry, 1897-1898: 249; Parodiz, 1946b: 358, 1957b: 24, 1962: 444; Lopes, 1957: 44; Figueiras, 1962: 47, 1963: 90; Shade, 1965: 217; Duarte, 1971: 84; Fernandez \& Castellanos, 1973: 280; Quintana, 1982: 97; Dutra-Clarke \& Souza, 1991: 293, pl. 2, figs. 1a-1b; Richardson, 1995: 159; Salgado \& Coelho, 2003: 162; Simone, 2006: 140, fig. 465; Cuezzo et al., 2013: 156; Salvador et al., 2018a: 112, figs. 7D-F; Salvador, 2019a: 86.

Drymaues (Mormus) papyraceus papyraceus: Morretes, 1949: 149.

Drymaeus papyraceus papyraceus: Figueiras, 1963: 90; Oliveira et al., 1972: 16; Oliveira \& Almeida, 1999: 36; Silva \& Castro, 2003: 67; Agudo-Padrón, 2008: 159, 2014: 15, fig. 10.

Type locality: Brazil, Rio de Janeiro.

Distribution: Widespread in Brazil, Paraguay, Argentina and Uruguay (Simone, 2006; Cuezzo et al., 2013).

Material studied: Brazil, Rio Grande do Sul: Itaara (29³6'S, 5345'W): 1 sh, iv.1982, UFSM, L. Indrusiak col. (MZSP 132587); Santa Maria (29 $\left.41^{\prime} \mathrm{S}, 5^{\circ} 48^{\prime} \mathrm{W}\right)$ : 1 sh, ix.1980, L. Indrusiak col. (MZSP 43338); 20 sh, iii.1990, UFSM, L. Indrusiak col. (MZSP 132558); 7 sh, xi.1990, UFSM, L. Indrusiak col. (MZSP 132559); 2 sh, x.1992, UFSM, S. Cechin col. (MZSP 132560); 5 sh, ix.1980, UFSM, L. Indrusiak col. (MZSP 132561); 1 sh, xi.1991, UFSM, S. Cechin col. (MZSP 132562); 1 sh, ii.1992, UFSM, L. Indrusiak col. (MZSP 132579).

\section{Drymaeus poecilus (d'Orbigny, 1835)} (Fig. 3K-L)

Helix (Cochlogena) poecila d'Orbigny, 1835: 11; Breure, 1979: 113.
Bulimus poecilus: d'Orbigny, 1837: 268, pl. 31, fig. 1-10; Gray, 1854: 15; Pfeiffer, 1848: 200; Reeve, 1849b: pl. 16, fig. 91.

Bulimus poecilus var. major d'Orbigny, 1837: 269.

Bulimus poecilus var. minor d'Orbigny, 1837: 269.

Bulimulus poecilus: Beck, 1837:65; Martens, 1881-1885: 13, fig. 1-5; Ancey, 1897: 5.

Bulimulus poecitus: Martens, 1895: 33.

Bulimus pictus Bonnet, 1864: pl. 5, figs. 4-6, pl. 6, fig. 1.

Bulimus poecilus var. icterica Ancey, 1892: 92.

Drymaeus poecilus: Pilsbry, 1897-1898: 285, pl. 49, figs. 49-57; Smith, 1903: 71; Morretes, 1943: 117; Parodiz, 1946b: 358, 1957b: 25, 1962: 440; Lopes, 1957: 44; Shade, 1965: 217; Breure, 1975b: 1152; Oliveira et al., 1992: 99; Richardson, 1995: 163; Oliveira \& Almeida, 1999: 37; Salgado \& Coelho, 2003: 162; Silva \& Castro, 2003: 67; Simone, 2006: 141, fig. 468; Cuezzo, et al., 2013: 156; Agudo-Padrón, 2014: 15; Salvador et al., 2015: 73, figs. 22-23, 2018b: 76, figs. 24-42; Birckolz et al., 2016, table 1; Salvador, 2019a: 86.

Drymaeus lynchi Parodiz, 1946a: 1, pl. 1, figs. 1-3.

Drymaeus (Drymaeus) poecilus: Breure \& Eskens, 1981: 32, fig. 102-108; Cuezzo \& Drahg, 1995: 193.

Drymaeus poecilus poecilus: Fernandez \& Castellanos, 1973: 280; Quintana, 1982: 95.

Drymaues (Leiostracus) poecilus: Oliveira \& Oliveira, 1984: 12; Morretes, 1949: 151.

Drymaeus poecila: Breure \& Ablett, 2014: 154, figs. 45M-N.

Type locality: Bolivia, Chiquitos province (see also Breure \& Ablett, 2014).

Distribution: Bolivia, Brazil (central to south), Paraguay, and Argentina (Salvador et al., 2018b).

Material studied: Brazil, Rio Grande do Sul: Santa Maria (29 $\left.41^{\prime} \mathrm{S}, 5^{\circ} 48^{\prime} \mathrm{W}\right)$ : 3 spm, 01.v.1984, D. Junges \& L. Indrusiak col. (MZSP 27662); 3 sh, ix.1979, D. Link col. (MZSP 43119); 2 sh, 04.vi.1990, UFSM, L. Indrusiak col. (MZSP 132482); 6 sh, ix.1993, UFSM, L. Indrusiak col. (MZSP 132580); 1 sh, vii.1990, UFSM, L. Indrusiak col. (MZSP 132591); 2 sh, ix.1980, UFSM, L. Indrusiak col. (MZSP 132582); 1 sh, xii.1994, UFSM. (MZSP 132583); 4 sh, ix.1980, UFSM, L. Indrusiak col. (MZSP 132584); 2 sh, viii.1980, UFSM, E. Loreto col. (MZSP 132585); 3 sh, ii.1992, UFSM, L. Indrusiak col. (MZSP 132586); (2 sh, ix.1979, UFSM, A. Alvarez col. (MZSP 132588): 2 sh, x.1993, UFSM, L. Indrusiak col. (MZSP 132592); 2 sh, iv.1994, UFSM, L. Indrusiak col. (MZSP 132593); 1 sh, v.1996, UFSM, S. Cechin col. (MZSP 132651); São Martinho da Serra

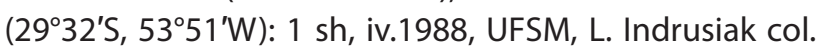
(MZSP 132590).

Remarks: The conchological features of this species are widely variable, regarding both shell shape and color pattern; the full range of variation was recently explored by Salvador et al. (2018b). 


\section{Family Odontostomidae Genus Cyclodontina Beck, 1837 Cyclodontina corderoi Klappenbach, 1958}

(Fig. 3M-P)

Cyclodontina (Spixia) corderoi Klappenbach, 1958: 2, pls. 1-2; Olazarri et al., 1972: 7; Breure, 1974: 114; Heydrich, 2016: 29.

Cyclodontina corderoi: Figueiras, 1963: 91; Breure \& Ablett, 2012: 11, figs. 21J-K, 21 iii.

Spixia corderoi: Scarabino, 2003: 209.

Type locality: Uruguay, Tacuarembó department, Tambores municipality, "Pozo Hondo”.

Distribution: Uruguay.

Material studied: Brazil, Rio Grande do Sul: Itaara (29³6'S, 5345'W), 37 sh, iv.1982, UFSM, L. Indrusiak col. (MZSP 132604); 4 sh, xi.1988, UFSM, L. Indrusiak col.

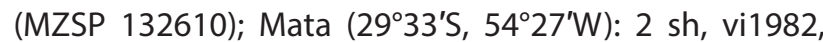
UFSM, M. Bier col. (MZSP 132619); Santa Maria (2941'S, 534' $\mathrm{W}$ ): 5 sh (MZSP 43133); 3 sh (MZSP 43136); 2 sh, ix.1980, L. Indrusiak col. (MZSP 43337); 5 sh, ix.1980, L. Indrusiak col. (MZSP 44146); 80 sh, ix.1980, UFSM, L. Indrusiak col. (MZSP 132603); 19 sh, vi.1979, UFSM, A. Fagundes col. (MZSP 132605); 20 sh, ii.1989, UFSM, L. Indrusiak col. (MZSP 132607); 1 sh, ii.1992, UFSM, L. Indrusiak col. (MZSP 132611); 1 sh, ix.1985, UFSM, P. Veiga col. (MZSP 132612); 1 sh, iii.1992, UFSM, L. Indrusiak col. (MZSP 132613); 1 sh, ix.1982, UFSM, L. Indrusiak col. (MZSP 132618); 2 sh, ix.1990, UFSM, L. Indrusiak col. (MZSP 132657); São Martinho da Serra (29³2'S, 535 $\left.51^{\prime} \mathrm{W}\right)$ : 6 sh, iv.1988, UFSM, L. Indrusiak col. (MZSP 132608); 2 sh, v.1990, UFSM, L. Indrusiak col. (MZSP 132615);

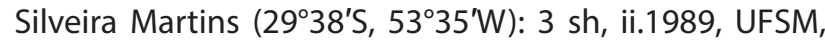
L. Indrusiak col. (MZSP 132609); 2 sh, xi.1978, UFSM, A. Fagundes col. (MZSP 132614).

Remarks: Cyclodontina corderoi can be identified by its shell shape, ochre coloration, the well-marked sinuous (sometimes anastomosed) axial sculpture of the teleoconch, and its aperture with four prominent teeth: a median parietal tooth, a median palatal tooth, a basal tooth, and a strong columellar lamella. A faint upper palatal tooth might also be present (Fig. 30). Furthermore, the overall shell shape can deviate a little from the typically conical spire (Fig. 3M) to very slender forms (Fig. 30), which are somewhat reminiscent of the genus Spixia Pilsbry \& Vanatta, 1898. The present record is the first report of this species from Brazil and increases its range circa $250 \mathrm{~km}$ to the north.

\section{Genus Macrodontes Swainson, 1840 Macrodontes gargantua (Férussac, 1821)} (Fig. 3Q-R)

Helix (Cochlogena) gargantua Férussac, 1821: 62; Breure, 1974: 110.
Bulimus odontostoma Sowerby, 1824: 59, pl. 5, fig. 3; Reeve, 1849b: pl. 38, fig. 228; Breure \& Ablett, 2012: 29, figs. 24A-D, $24 \mathrm{i}$.

Odontostomus gargantula: Beck, 1837: 54.

Macrodontes sowerbeyii: Swainson, 1840: 334.

Macrodontes odontostomus: Pilsbry, 1901-1902: 31, pl. 13, figs. 85-87; Pfeiffer, 1855: 149.

Macrodontes jöergensenianus Holmberg, 1912: 153, figs. 11-13.

Odontostomus bertonii Ihering in litt.: Bertoni, 1926: 72 [nomem nudum].

Macrodontes thielei Pilsbry, 1930b: 360, pl. 31, fig. 1.

Macrodontes odontostoma: Morretes, 1949: 157.

Odontostomus gargantua: Breure \& Schouten, 1985: 22; Breure \& Ablett, 2012: 30, figs. 24A-D, 24i; Cuezzo et al., 2013: 165.

Odontostomus (Odontostomus) gargantua: Quintana, 1982: 98.

Odontostomus (Euodontostomus) joergensenianus: Breure, 1974: 117.

Odontostomus odontostomus joergensenianus: Parodiz, 1957b: 29; Fernandez \& Castellanos, 1973: 280.

Macrodontes gargantua: Pfeiffer, 1842: 136; Simone, 2006: 162, fig. 155; Agudo-Padrón, 2008: 163, 2014: 18; Gregoric et al., 2013: 1763; Breure \& Araujo, 2017: 51, fig. 22G.

Type locality: Undefined.

Distribution: Brazil (Santa Catarina state) and Argentina (Missiones province) (Simone, 2006).

Material studied: Brazil, Rio Grande do Sul: Santa Maria

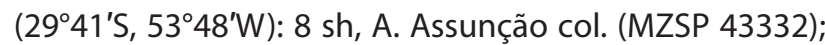

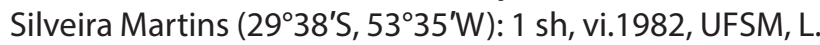
Indrusiak col. (MZSP 132652).

Remarks: The slender profile of the body whorl, the oval aperture, the raised ridge on the abapical portion of the body whorl close to the aperture, and the number and position of the teeth (in particular the shape of the upper columellar lamella), are all consistent with M. gargantua. The present record extends the species distribution to the southeast.

\section{Family Simpulopsidae Genus Simpulopsis Beck, 1837 Simpulopsis sulculosa (Férussac, 1821)} (Fig. 3S-T)

Helix (Cochlohydra) sulculosa Férussac, 1821: 31, pl. 11A, fig. 6; Moricard, 1836: 415; Breure, 1979: 134.

Vitrina sulculosa: Hupé, 1857: 4.

Simpulopsis (Simpulopsis) sulculosa: Morretes, 1949: 161; Haas, 1953: 205; Breure, 1973: 52, 1975a: 97, 1975b: 1157.

Simpulopsis sulculosa: Gray, 1825: 415; Pfeiffer, 1841: 74; Reeve, 1862: pl. 2, fig. 13; Heynemann, 1868: 110; Martens, 1868: 183; Hidalgo, 1869: 5, 1870: 30; 
Clessin, 1888: 168; Pilsbry, 1899: 214, pl. 64, fig. 81-83; Richardson, 1995: 367; Oliveira \& Almeida, 1999: 41; Salgado \& Coelho, 2003: 164; Silva \& Castro, 2003: 67; Simone, 2006: 180, fig. 648; Agudo-Padrón, 2008: 160; Santos et al., 2010: 513; Birckolz et al., 2016: table 1; Breure \& Araujo, 2017: 90, fig. 33H; Salvador et al., 2018a: 114, figs. 9F-G; Salvador, 2019a: 89.

\section{Type locality: Brazil.}

Distribution: Brazil, from Alagoas state to Rio Grande do Sul state (Salvador et al., 2018a).

Material studied: Brazil, Rio Grande do Sul: Agudo (29³8'S, 5315'W): 1 sh, x.1988, UFSM, L. Indrusiak col. (MZSP 132663); Santa Maria (29 $\left.41^{\prime} \mathrm{S}, 53^{\circ} 48^{\prime} \mathrm{W}\right)$ : 2 sh, 07.vi.1996, S. Cechin col. (MZSP 132522); 1 sh, xii.1990, UFSM, L. Indrusiak col. (MZSP 132660); 7 sh, x.1992, UFSM, S. Cechin col. (MZSP 132661); 1 sh, vi.1990, UFSM, L. Indrusiak col. (MZSP 132662).

\section{Superfamily Pupilloidea Family Valloniidae Genus Pupisoma Stoliczka, 1873 Pupisoma dioscoricola (Adams, 1845)}

(Fig. 3U-V)

Helix discoricola Adams, 1845: 16.

Pupisoma dioscoricola: Pilsbry, 1920-1921: 36, pl. 4, figs. 1-5; Fernandez \& Castellanos, 1973: 270; Quintana, 1982: 73; Cuezzo \& Drahg, 1995: 188; Salgado \& Coelho 2003: 153; Rumi et al., 2010: 2988; Agudo-Padrón, 2014: 18; Salvador et al., 2017: 139, fig. 12, 2018a: 109, fig. 6F.

Pupisoma discoricola [sic]: Simone, 2006: 308, fig. 9.

Pupisoma (Ptychopatula) dioscoricola: Hausdorf, 2007: 1483, figs. 1-2.

Type locality: USA, Texas, Brownsville.

Distribution: From Florida, USA, to northern Argentina and southern Brazil, including the Caribbean islands (Hausdorf, 2007).

Material studied: Brazil, Rio Grande do Sul: Santa Maria (2941'S, 5348'W): 3 sh, ii.1992, UFSM, L. Indrusiak col. (MZSP 132669); 15 sh, vi.1990, UFSM, L. Indrusiak col. (MZSP 132671); 5 sh, iv.1991, UFSM, L. Indrusiak col. (MZSP 132672); São Martinho da Serra (29³2'S, $53^{\circ} 51^{\prime} \mathrm{W}$ ): 1 sh, v.1990, UFSM, L. Indrusiak col. (MZSP 132670).

Remarks: Despite the species being known throughout the Americas, the present record is on the southernmost edge of the species distribution (see Hausdorf, 2007: fig. 6). Santa Maria is just $10 \mathrm{~km}$ south of the southernmost record reported by Hausdorf (2007): Taquara municipality, also in Rio Grande do Sul state.

\section{Superfamily Trochomorphoidea Family Euconulidae \\ Genus Pseudoguppya Baker, 1925 \\ Pseudoguppya semenlini (Moricand, 1846)}

(Fig. 4A-C)

Helix Semen-lini Moricand, 1846: 149, pl. 5, fig. 17; Pfeiffer, 1846: 457, 1848: 31, 1853: 32; Reeve, 1854: pl. 112, fig. 637; Hupé, 1857: 14.

Helix (Hyalina) semen lini: Albers, 1860: 73.

Hyalina semen lini: Heynemann, 1868: 106; Clessin, 1888: 166.

Hyalina (Conulus) semen lini: Martens, 1868: 175.

Conulus semen lini: Ihering, 1894: 38.

Guppya semen-lini: Ancey, 1897: 9.

Guppya seminlini: Pilsbry, 1900: 386.

Vitrea semen lini: Suter, 1900: 331.

Habroconus (Pseudoguppya) semen-lini: Haas, 1953: 205, 1959: 365.

Habroconus (Pseudoguppya) semenlini: Baker, 1928: 12; Morretes, 1949: 137; Figueiras, 1963: 87; Fernandez \& Castellanos, 1973: 274; Quintana, 1982: 79.

Habroconus semenline: Salgado \& Coelho, 2003: 154.

Habraconus semenlini: Klappenbach, 1967: 42; AgudoPadrón, 2008: 166, 2014: 18; Santos et al., 2010: 516.

Pseudoguppya semenlini: Salvador et al., 2016: 65, 2018b: 124, figs. 14A-C; Salvador, 2019a: 95.

Type locality: Brazil, Bahia state.

Distribution: Brazil (Alagoas, Bahia, Rio de Janeiro, São Paulo, Paraná, Santa Catarina, and Rio Grande do Sul states), Paraguay, Uruguay, Argentina (Salvador et al., 2018b).

Material studied: Brazil, Rio Grande do Sul: Santa Maria (2941'S, 5348'W): 5 spm, 04.ii.1992, UFSM, L. Indrusiak col. (MZSP 132541); 1 spm, 06.vii.1990, UFSM, L. Indrusiak col. (MZSP 132542); 3 spm, 16.i.1992, UFSM, S. Cechin col. (MZSP 132543).

\section{Superfamily Gastrodontoidea Family Gastrodontidae Genus Zonitoides Lehmann, 1862 Zonitoides sp. \\ (Fig. 4D-F)}

Material studied: Brazil, Rio Grande do Sul: Santa Maria (2941'S, 534' W): 1 sh, v.1982, L. Indrusiak col. (MZSP 43137).

Remarks: Zonitoides species are distributed through North America and Eurasia, but some have been introduced elsewhere. In Brazil, there are reports of two species: Z. nitidus (Müller, 1774) and Z. arboreus (Say, 1817) (Simone, 2006). The present specimen is an empty shell and conchological characters alone are not sufficient for a confident identification at species-level. 


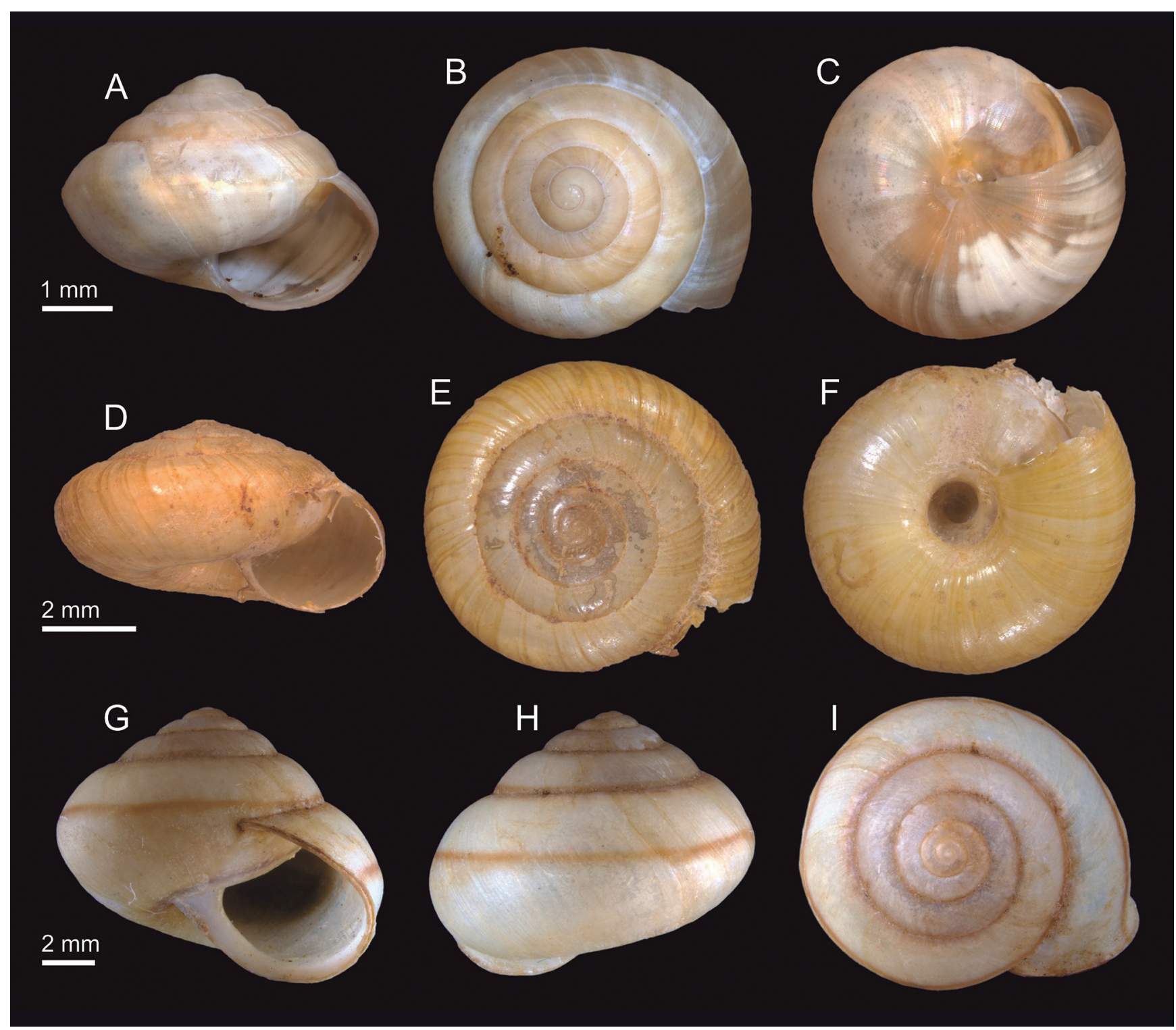

Figure 4. $(A-I)=$ Shells from Santa Maria region, Rio Grande do Sul state. $(A-C)=$ Pseudoguppya semenlini MZSP $132543(L=3.4 \mathrm{~mm}, D=4.5 \mathrm{~mm})$. $(D-F)=$ Zonitoides sp. MZSP $43137(\mathrm{~L}=4.9 \mathrm{~mm}, \mathrm{D}=8.2 \mathrm{~mm}) .(\mathrm{G}-\mathrm{I})=$ Bradybaena similaris MZSP $132575(\mathrm{~L}=10.6 \mathrm{~mm}, \mathrm{D}=12.5 \mathrm{~mm})$.

\section{Superfamily Helicoidea \\ Family Camaenidae \\ Genus Bradybaena Beck, 1837 \\ Bradybaena similaris (Férusac, 1821)}

(Fig. 4G-I)

Helix similaris Férussac, 1821: 43; Férussac \& Deshayes, 1819-1851: 171, pl. 25, 27; d'Orbigny, 1835: 7; Gray, 1854: 11; Reeve, 1854: pl. 34; Martens, 1868: 175.

Helix (Fruticicola) similaris: Clessin, 1888: 166.

Bradybaena similaris: Morretes, 1949: 165; Haas,

1955: 373; Pitoni et al., 1976: 27, fig. 2; Quintana,

1982: 106; Araujo, 1989: 586, figs. 1-10; Boffi, 1989: 39,

fig. 99; Picoral \& Thomé, 1989: 435, figs. 1-6; Santos, 1994: 376, fig. 1; Oliveira \& Almeida, 1999: 49; Simone, 1999: 5, 2006: 312, fig. 29; Bruschi-Figueiró \& Veitenheimer-Mendes, 2002: 32; Salgado \& Coelho, 2003: 174; Miquel et al., 2007: 114; Agudo-Padrón, 2008: 163, 2014: 18; Rumi et al., 2010: 2987; AgudoPadrón \& Lenhard, 2011: 169

\section{Type locality: Timor.}

Material studied: Brazil, Rio Grande do Sul: Santa Maria ( $29^{\circ} 41^{\prime}$ 'S, 53ํํ' W): 5 sh, iv. 1992, UFSM, D. Linck col. (MZSP 132575); 1 sh, UFSM, M. Krügel col. (MZSP 132576).

Remarks: This species is native of Southeast Asia and has been introduced in many parts of the world. In Brazil, it is known to occur throughout most of the country's states, from the Equatorial region to the southernmost states (Araujo, 1989; Junqueira et al., 2003).

\section{DISCUSSION}

Of the 20 species reported herein, four represent significative extensions of geographic distribution, namely Adelopoma brasiliense, Happia vitrina, Macrodontes gargantua, and Cyclodontina corderoi, the latter being the first record of the species in Brazil. 
This is not surprising, as the terrestrial molluscan fauna of Brazil has been scarcely studied in some regions (Salvador, 2019b). Only now, little by little, we are starting to get a better view of species diversity and distribution. Still, given the size of the country, it is expected that several localities remain un- or undersampled. In this regard, local collections, such as UFSM's studied here, are of utmost importance, as they can bring to light specimens that otherwise would have been overlooked.

The specimens give a reasonable idea of the land snail fauna that can be found in the region. However, they were collected two or three decades ago, so some might not be present there anymore; much has inevitably changed as the cities grow larger and more areas open way to agriculture, so the current status of these species in the region must be further studied. It is also worthwhile to mention that older collections inevitably focus on larger and more aesthetically pleasing specimens, which largely ignores the diversity of microsnails (Salvador, 2019b), as can be seen here by the lack of typically small but abundant groups such as the Punctoidea.

The region of Santa Maria represents the transition between two main Brazilian biomes: the Atlantic Forest and the Pampas (Löbler et al., 2015). Such transitional areas often comprehend a very diverse molluscan fauna, with representatives of both biomes. This can be seen in the present assembly of species, with species known from the Atlantic Forest areas of Brazil and others known from the Pampas of Uruguay and Argentina.

Two introduced species were found in the studied material: Bradybaena similaris and Zonitoides sp. Despite being commonly found in Brazil, the presence of these exotic species hints about the state of conservation efforts in the region. There are conservation areas and reserves on the region (e.g., Quarta Colônia State Park in Agudo and Ibarama municipalities), but their actual state can be troubling. In Santa Maria, for instance, there are several protected areas delimited on paper, but most are occupied, and the law is not enforced (Nascimento \& Foleto, 2011).

\section{ACKNOWLEDGEMENTS}

We are very grateful to Dr. Carla B. Kotzian for kindly donating the UFSM collection to the MZSP; to José $\mathrm{H}$. Fontenelle for confirming our identification of Megalobulimus spp.; to Leocádia Indrusiak and C.B. Kotzian for the help in sorting out the collection localities; and to the anonymous reviewers for their comments and suggestions to improve the article. This study was partly financed by the Coordenação de Aperfeiçoamento de Pessoal de Nível Superior - Brasil (CAPES) - Finance Code 001. RBS acknowledges the bequest of Bruce Fraser Hazelwood and the Museum of New Zealand Te Papa Tongarewa.

\section{REFERENCES}

Achaval, M.; Penha, M.A.P.; Swarowsky, A.; Rigon, P.; Xavier, L.L.; Viola, G.G. \& Zancan, D.M. 2005. The terrestrial Gastropoda Megalobulimus abbreviatus as a useful model for nociceptive experiments. Effects of morphine and naloxone on thermal avoidance behavior. Brazilian Journal of Medical and Biological Research, 38: 73-80.

Adams, C.B. 1845. Specierum novarum conchyliorum, in Jamaica reper-torum, synopsis. Proceedings of the Boston Society of Natural History, 2: 1-86.

Agudo-Padrón, A.l. 2008. Listagem sistemática dos moluscos continentais ocorrentes no estado de Santa Catarina, Brasil. Comunicaciones de la Sociedad Malacológica del Uruguay, 9(91): 147-179.

Agudo-Padrón, A.I. 2014. Inventario sistemático de los moluscos continentales ocurrentes en el Estado de Santa Catarina, Brasil. BIOMA, 2(21): 6-23.

Agudo-Padrón, A.I. 2015. Molluscs of Santa Catarina State, SC, Central Southern Brazil: increments to species inventory, new geographical records and additional informations. International Journal of Aquaculture, 5(2): 1-8.

Agudo-Padrón, A.I. \& Lenhard, P. 2011. Continental mollusc fauna of the Great Porto Alegre central region, RS, Southern Brazil. Biodiversity Journal, 2(4): 163-170.

Albers, J.C. 1860. Die Heliceen. Leipzig, Verlag von Wilhelm Engelmann. 359 p. Altena, C.O.v.R. 1960. On small collection of land Mollusca from Surinam (Dutch Guiana). Basteria, 24(4): 48-51.

Ancey, C.F. 1892. On some shells from eastern Bolivia and western Brazil. Journal of Conchology, 7(3): 90-97.

Ancey, C.F. 1897. Viaggio del Dott. Alfredo Borelli nel chaco boliviano e nella Repubblica Argentina XI. Resultats malacologiques accompagnés d'une notice sur les espèces précédemment recueillies par ce voyageur. Bollettino dei Musei di Zoologia ed Anatomia Comparada dela R. Universitá diTorino, 12(309): 1-22.

Araujo, J.L.B. 1989. Moluscos de importância econômica no Brasil. I. Xanthonychidae: Bradybaena similaris (Férussac, 1821), (Mollusca, Gastropoda, Pulmonata, Stylommatophora). Revista Brasileira de Zoologia, 6(4): 583-592.

Araujo, J.L.B. \& Keller, D.G. 1993. Moluscos de importância econômica no Brasil. iii. Subullnidae, Leptinaria unllamellata (Orbigny) (Mollusca, Gastropoda, Pulmonata, Stylommatophora). Revista Brasileira de Zoologia, 10(3): 499-507.

Arias, S. 1953. Algunos moluscos de la region de Perija. Memoria de la Sociedad de Ciências Naturales la Salle, Caracas, 15(35): 245-257.

Baker, F. 1913. The land and freshwater mollusks of Standford Expedition to Brazil. Proceedings of the Academy of Natural Sciences of Philadelphia, 65: 618-672, pls. 21-27.

Baker, H.B. 1927. The Mollusca collected by the University of Michigan Williamson Expedition in Venezuela. Part V. Occasional Papers of the Museum of Zoology, University of Michigan, 8(182): 1-36.

Baker, H.B. 1928. Minute American Zonitidae. Proceedings of the Academy of Natural Sciences of Philadelphia, 80: 1-44.

Baker, H.B. 1945. Some American Achatinidae. Nautilus, 58(3): 84-92.

Barattini, L.P. \& Ledón, D.A. 1949. Uma nueva subespecie del gênero Strophocheius para la malaco-fauna uruguaya. Revista de la Sociedad Malacologica "Carlos de la Torre", 7(1): 1-2.

Beck, H.H. 1837-1838. Index molluscorum praesentis aevis Musei Principis augustissimi Christiani Frederici. Fasciculus primus et secundus Mollusca gastraeopoda pulmonata. Hafniae. 124 p. +8 p. [append.].

Bequaert, J.C. 1948. Monograph of the Strophocheilidae, a neotropical family of terrestrial mollusks. Bulletin of the Museum of Comparative Zoology, Cambridge, 100(1): 3-210, pls. 1-32.

Bertoni, A.W. 1926. Sobre moluscos del Paraguay. Revista de la Sociedad Cientifica del Paraguay, 2(1): 71-73.

Birckolz, C.J.; Salvador, R.B.; Cavallari, D.C. \& Simone, L.R.L. 2016. Illustrated checklist of newly described (2006-2016) land and freshwater Gastropoda from Brazil. Archiv für Molluskenkunde, 145(2): 133-150. 
Boffi, A.V. 1989. Moluscos brasileiros de interesse médico e econômico. São Paulo, Ed. Hucintec; FAPESP. $182 \mathrm{p}$.

Bonnet, A. 1864. Description de quelques mollusques nouveaux ou présumé tels. Revue et Magasin de Zoologie Pure et Appliquée, 26: 67-72.

Borda,V.\&Ramírez, R. 2013. Re-characterization of the Red-lip Megalobulimus (Gastropoda: Strophocheilidae) from Peru with description of a new species. Zoologia, 30(6): 675-691.

Bouchet, P.; Rocroi, J.P.; Hausdorf, B.; Kaim, A.; Kano, Y.; Nützel, A.; Parkhaev, P.; Schrödl, M. \& Strong, E.E. 2017. Revised classification, nomenclator and typification of gastropod and monoplacophoran families. Malacologia, 61(1-2): 1-526.

Breure, A.S.H. 1973. Catalogue of Bulimulidae (Gastropoda, Euthyneura), I. Amphibulimulidae. Basteria, 37: 51-56.

Breure, A.S.H. 1974. Catalogue of Bulimulidae (Gastropoda, Euthyneura), II. Odontostominae. Basteria, 38: 109-125.

Breure, A.S.H. 1975a. Notes on Bulimulidae (Gastropoda, Euthyneura), 2. On a small collection of Simpulopsis Beck, 1837, from southern Brazil, with description of three new species. Basteria, 39: 97-113.

Breure, A.S.H. 1975b. Types of Bulimulidae (Mollusca, Gastropoda) in the Muséum National d'Histoire Naturelle, Paris. Bulletin du Muséum National d'Histoire Naturelle, 3. Ser. 331, Zool. 233: 1137-1187.

Breure, A.S.H. 1979. Systematics, phylogeny and zoogeography of Bulimulinae (Mollusca). Zoologische Verhandelingen, Leiden, 168: 1-215.

Breure, A.S.H. \& Ablett, J.D. 2012. Annotated type catalogue of the Bothriembryontidae and Odontostomidae (Mollusca, Gastropoda, Orthalicoidea) in the Natural History Museum, London. Zookeys, 182: 1-70.

Breure, A.S.H. \& Ablett, J.D. 2014. Annotated type catalogue of the Bulimulidae (Mollusca, Gastropoda, Orthalicoidea) in the Natural History Museum, London. Zookeys, 392: 1-367.

Breure, A.S.H. \& Ablett, J.D. 2015. Annotated type catalogue of the Megaspiridae, Orthalicidae, and Simpulopsidae (Mollusca, Gastropoda, Orthalicoidea) in the Natural History Museum, London. Zookeys, 470: 17-143.

Breure, A.S.H. \& Araujo, R. 2017. The Neotropical land snails (Mollusca, Gastropoda) collected by the 'Comisión Científica del Pacífico'. PeerJ, 5: e3065.

Breure, A.S.H. \& Eskens, A.A.C. 1981. Notes on and descriptions of Bulimulidae (Mollusca, Gastropoda), II. Zoologische Verhandelingen Leiden, 186: $1-111$.

Breure, A.S.H. \& Schouten, J.R. 1985. Notes on and descriptions of Bulimulidae (Mollusca, Gastropoda), III. Zoologische Verhandelingen, 216: 3-98.

Bruschi-Figueiró, G. \& Veitenheimer-Mendes, I.L. 2002. Moluscos em área de horticultura no município de Porto Alegre, Rio Grande do Sul, Brasil. Revista Brasileira de Zoologia, 19(2): 31-37.

Buckup, L. \& Buckup, E.H. 1957. Catálogo dos moluscos do Museu RioGrandense de Ciências Naturais. Iheringia Ser. Zoologia, 1: 1-40.

Capinera, J.L. 2017. Biology and food habits of the invasive snail Allopeas gracile (Gastropoda: Subulinidae). Florida Entomologist, 100(1): 116-123.

Clessin, S. 1888. Binnenmollusken aus Südbrasilien. Malakozoologische Blätter, 10(2): 165-174.

Cuezzo, M.G. \& Drahg, F. 1995. Moluscos depositados en la Colección de la Fundación Miguel Lillo: Argentina. Acta zoológica Lilloana, 43(1): 185-205.

Cuezzo, M.G.; Miranda, M.J. \& Ovando, X.M.C. 2013. Species catalogue of Orthalicoidea in Argentina (Gastropoda: Stylommatophora). Malacologia, 56: 135-191.

d'Orbigny, A. 1834-1847. Voyage dans l'Amérique Méridionale. Mollusqués, Paris, Piotois Levrault. v. 5, pt. 3,758 p. +85 pls.

d'Orbigny, A. 1835. Synopsis terrestrium et fluviatilum molluscorum, in suo per American Meridionalem itinerere. Magasin de Zoologie, 5(6): 1-44.
Delannoye, R.; Charles, L.; Pointier, J.P. \& Massemin, D. 2015. Mollusques continentaux de la Martinique. Paris, Muséum national d'Histoire Naturelle. $328 \mathrm{p}$.

Doering, A. 1874. Apuntamientos sobre la fauna de moluscos de la Republica Argentina. Boletín de la Academia Nacional de Ciencias em Cordoba, Argentina, 1: 48-77.

Duarte, E. 1971. Lo nuestro em "Los moluscos del viaje al Pacifico" de Hidalgo y Martinez. Comunicaciones de la Sociedad Malacológica del Uruguay, 20(3): 83-89.

Dutra, A.V.C. 1988. Aspectos da ecologia e da reprodução de Leptinaria uniamellata (Orbigny, 1835) (Gastropoda, Subullnidae). Revista Brasileira de Zoologia, 5(4): 581-591.

Dutra-Clarke, A.V.C. \& Souza, F.B.V.A. 1991. Bumulidae (Gastropoda, Stylommatophora) do Nordeste do Brasil. Revista Brasileira de Zoologia, 7(3): 289-304.

Fernandez, D. \& Castellanos, Z.J.A. 1973. Clave generica de la malacofauna terrestre argentina. Revista del Museo de la Plata, 11(107): 265-285.

Férussac, A.E.J.P.J.F.A. 1819. Histoire naturelle des pülmonés sans opercule. Genre Bulime. In: Histoire naturelle generale et particuliére des mollusques terrestres et fluviatiles.... Paris. 128 p. ilus +32 p. +2 v. atlas.

Férussac, A.E.J.P.J.F.A. 1821. Tableaux systématiques des animaux mollusques classés en familles naturelles, dans lesquels on a établi la concordance de tous les systèmes; suivis d'un prodrome général pour tous les mollusques terrestres ou fluviatiles, vivants ou fossilles. Paris, Arthus Bertrand.

Férussac, A.E.J.P.J.F.A. \& Deshayes, G.P. 1819-1851. Histoire naturelle générale et particuliére des mollusques terrestres et fluviatiles. Paris, Chez J.B. Baillière. $868 \mathrm{p}$.

Figueiras, A. 1962. Sobre la presencia del género Protoglyptus Pilsbry em el Uruguay (Bulimulinae). Comunicaciones de la Sociedad Malacologica del Uruguay, 1(2): 47-48.

Figueiras, A. 1963. Enumeracíon sistemática de los moluscos terrestres del Uruguay. Comunicaciones de la Sociedad Malacologica del Uruguay, 1(4): 79-96.

Fontenelle, J.H.; Cavallari, D.C. \& Simone, L.R.L. 2014. A new species of Megalobulimus (Gastropoda, Strophocheilidae) from Brazilian shell mounds. Strombus, 21(1-2): 30-37.

Fontenelle, J.H.; Tomotani, B.M. \& Salvador, R.B. 2019. Taxonomic reassessment of Megalobulimus toriii (Gastropoda, Strophocheilidae). Journal of Conchology, 43(3): 313-320.

Götting, K.L. 1978. Lista preliminar de los caracoles terrestres de la region septentrional de Colombia. Anales del Instituto de Investigaciones Marinas de Punta de Betin, 10: 101-110.

Gray, J.E. 1825. A list and description of some species of Shells not taken Notice of by Lamarck. Annals of Philosophy, new series, 9: 407-415.

Gray, J.E. 1847. A list of the genera of recent Mollusca, their synonima and types. Proceedings of the Zoological Society of London, 15: 129-219.

Gray, J.E. 1854. List of the shells of South America in the collection of the British Museum collected and described by Alcide d' Orbigny in the "Voyage dans I'Amérique Méridionale.' London. $89 \mathrm{p}$.

Gregoric, D.E.G.; Núñes, V.; Vogles, R.E.; Beltramino, A.A. \& Rumi, A. 2013. Gasterópodos terrestres de la provincia de Misiones, Argentina. Revista de Biología Tropical, 61(4): 1759-1768.

Gude, G.K. 1902. A synopsis of the genus Streptaxis and its allies. Proceedings of the Malacological Society of London, 5(3): 201-244.

Haas, F. 1935. Kurze bemerkungen V (3): binnenschnecken aus verschiedenen teilen Brasiliens. Archiv für Molluskenkunde, 67(3): 107-112.

Haas, F. 1939. Zurkenntnis der binnen-Mollusken N.0.-Brasilien. Senckenbergiana, 21(3-4): 254-278.

Haas, F. 1953. Mollusks from Ilha Grande, Rio de Janeiro, Brazil. Fieldiana (Zoology), 34(20): 203-209. 
Haas, F. 1955. On some small collections of inland shells from South America. Fieldiana (Zoology), 34(35): 361-387.

Haas, F. 1959. Inland mollusks from Venezuela, southern Brazil and Peru. Fieldiana (Zoology), 39(31): 363-371.

Hausdorf, B. 2007. Revision of the American Pupisoma species (Gastropoda: Pupilloidea). Journal of Natural History, 41(21-24): 1481-1511.

Heydrich, I. 2016. Catálogo do material-tipo depositado nas coleções malacológicas do museu de Ciências Naturais da Fundação Zoobotânica do Rio Grande do Sul. Arquivos de (iências do Mar, 49(supl): 26-36.

Heynemann, D.F. 1868. Die Mundtheile einiger brasilianischen land-und süsswasserschnecken. Malakozoologische Blätter, 15: 99-113.

Hidalgo, J.G. 1869. Moluscos del viaje al Pacífico: verificado de 1862 á 1865 por una Comision de Naturalistas enviada por el Gobierno Español. Parte primeira univalvos terrestres. Madrid, Miguel Ginesta. 152 p.

Hidalgo, J.G. 1970. Catalogue des coquilles terrestres recueillies par naturalists de la commission scientifique espagnole sur divers points de l'Amérique méridionale. Journal de Conchyliologie, 18: 27-70.

Holmberg, E.L. 1912. Bulimuli et odontostomi argentini adhuc indescripti, necnon species ad subgenus nondum relatae. Anales del Museo Argentino de Ciencias Naturales "Bernardino Rivadavia", 23: 147-153.

Horn, A.C.M.; Achaval, A. \& Zancan, D.M. 2005. The annual reproductive cycle of the snail Megalobulimus abbreviatus (Bequaert, 1948) (Gastropoda, Pulmonata). Brazilian Journal of Biology, 65(3): 459-467.

Hupé, H. 1857. Mollusques. In: Castelnau, F. (Ed.). Animaux nouveaux ou rares recueillis pendant l'expédition dans les parties centrales de l'Amérique du Sud, de Rio de Janeiro à Lima, et de Lima au Para; par ordre gouvernement Français pendant les années 1843 à 1847, Paris, Bertrand. Partie 7, tome 3, pt. 3. 103 p.

Hutton, T. 1834. On the Land Shells oflndia. Journal of the Asiatic Society of Bengal, 26(6): 81-93.

Ihering, H. 1884. Über den Uropmeustichen apparat der Heliceen. Zeitschrift für wissenschaftliche Zoologie, 41: 259-283.

Ihering, H. 1894. Uber binnen-conchylien der küstenzone von Rio Grande do Sul. Archiv für Naturgeschichte, 60: 37-40.

Jaeckel, S. 1927. Die mollusken der müllegger'schen Brasilenexpedition. Zoologischer Anzeiger, 72(5-8): 129-139.

Jaeckel, S. 1952. Short review of the land and freshwater molluscs of the Nort-East States of Brazil. Dusenia, 3(1): 1-10.

Junqueira, F.0.; D'ávila, S.; Bessa, E.C.A. \& Prezoto, F. 2003. Ritmo de atividade de Bradybaena similaris (Férussac, 1821) (Mollusca, Xanthonychidae) de acordo com a idade. Revista de Etologia, 5(1): 41-46.

Klappenbach, M.A. 1958. Una nueva especie de Cyclodontina del Uruguay (Gastr. Pulm.). Comunicaciones Zoologicas del Museo de Historia Natural de Montevideo, 4(81): 1-4.

Klappenbach, M.A. 1967. La primeira lista de moluscos publicada em el Uruguay. Comunicaciones de la Sociedad Malacologica del Uruguay, 2(12): 41-44.

Kobelt, W. 1906. Die Raublungenschnecken (Agnatha). In: Martini, F.H.W. \& Chemnitz, J.H. (Eds.). Systematisches Conchylien-Cabinet, Nürnberg, Bauer \& Raspe. v. 12b, pt. 2.211 p.

Küster, H.C. 1850. Die Bulimiden und Achatinen In: Martini \& Chemnitz. Systematisches Conchylien-Cabinet. Nürnberg, Bauer \& Raspe. v. 13, pt. 1. $395 \mathrm{p}$.

Lamarck,J.B.P.A.M. 1822. Histoirenaturelle des animaux sansvertèbres, presentant les caractères généraux et particuliers de ces animaux précédée d'une introduction offrant la détermination des caractères essentiels de l'animal, sa distinction du végétal et des autres corps naturels, enfin, l'exposition des principes fondamentaux de la zoologie. Paris, Chez L'Auteur. v. 6, pt. 2, 232 p.

Lanzieri, P.D. 1966. A família Succineidae (Gastropoda, Pulmonata) da Ilha de Trindade, Costa do Sul. Papéis Avulsos do Departamento de Zoologia, 19(14): 169-188.
Leme, J.L.M. 1973. Anatomy and systematics of the neotropical Strophocheiloidea (Gastropoda, Pulmonata) with the description of a new family. Arquivos de Zoologia de São Paulo, 23(5): 295-337.

Löbler, C.A.; Sccoti, A.A.V. \& Werlang, M.K. 2015. Contribuição à delimitação dos biomas Pampa e Mata Atlântica no município de Santa Maria, RS. Revista Eletrônica em Gestão, Educação e Tecnologia Ambiental, 19(2): 1250-1257.

Lopes, H.S. 1957. Relação dos moluscos coletados na excursão às zonas das estradas de ferro Noroeste do Brasil e Brasil-Bolívia, nos estados de São Paulo e Mato Grosso, Brasil e Bolívia em janeiro e fevereiro de 1955. Publicações Avulsas do Museu Nacional, 20: 43-44.

Marshall, B.A. 1996. Molluscan name-bearing types in the Museum of New Zealand Te Papa Tongarewa. Tuhinga, 9: 1-85.

Martens, E.v. 1868. Ueber südbrasilianische land-und süsswassermollusken. Malakozoogische Blätter, 15: 169-217.

Martens, E.v. 1873. Die binnenmolluskenVenezuela's. In:FertschriftzurFeierdes hundertgähringen Bestehens. Berlin, Gesellschaft Naturfoschender Freunde zu Berlin. p. 157-225.

Martens, E.v. 1881-1885. Conchologische mittheilungen als fortsetzung der novitates conchologicae. Cassel, Verlag von Theodor Fischer. $19 \mathrm{p}$.

Martens, E.v. 1895. Mollusken von Paraguay. Sitzungsbenichte der Gesellschaft Naturforschender Freunde zu Berlin, 3: 33-35.

Martins, C.M. \& Simone, L.R.L. 2014. A new species of Adelopoma from são paulo urban park, Brazil (Caenogastropoda, Diplommatinidae). Journal of Conchology, 41(6): 766-773.

Massemin, D.; Lamy, D.; Pointier, J.P. \& Gargominy, 0. 2009. Coquillages et escargots de Guyane. Paris, Muséum national d'Histoire naturelle. $456 \mathrm{p}$.

Mawe, J. 1823. The Linnaean system of conchology, describing the orders, genera, and species of shells. London. $207 \mathrm{p}$.

Miquel, S.E. 1991. El género Bulimulus Leach, 1814 (Mollusca, Gastropoda, Stylommatophora) en la Republica Argentina. Studies on Neotropical Fauna and Environment, 26: 93-112.

Miquel, S.E. \& Aguirre, M.L. 2011. Taxonomía de los gasterópodos terrestres del Cuaternario de Argentina. [Taxonomy of terrestrial gastropods from the Quaternary of Argentina]. Revista Española de Paleontología, 26(2): 101-133.

Miquel, S.E.; Tablado, A. \& Sodor, A. 2007. Curaduría en la colección nacional de invertebrados de argentina: aportes a la biodiversidad y biogeografía de gasterópodos terrestres argentinos. Comunicaciones de la Sociedad Malacológica del Uruguay, 9: 113-115.

Miquel, S.E.; Turienzo, P.\& Di lorio, 0.R. 2015. Gastropod species found in birds' nests from Argentina. Revista del Museo Argentino de Ciencias Naturales, 17(1): 87-96.

Moricard, S. 1836. Mémorie sur les coquilles terrestres et fluviatiles, envoyées de Bahia par M.J. Blanchet. Memoires de la Société de Physique et d'Histoire Naturelle de Genève, 7(2): 415-446.

Moricand, S. 1846. Troisième supplément au mémoire sur les coquilles terrestres et fluviatiles de la province de Bahia, envoyées par M. Blanchet. Memoires de la Société de Physique et d'Histoire Naturelle de Genève, 11: 147-160.

Morretes, F.L. 1943. Contribuição ao estudo da fauna brasileira de moluscos: resultados de uma pequena coleção de moluscos obtida pela excursão científica realizada pelo Instituto Osvaldo Cruz em outubro de 1938. Papéis Avulsos do Departamento de Zoologia, 3(7): 111-126.

Morretes, F.L. 1949. Ensaio de Catálogo dos Moluscos do Brasil. Arquivo do Museu Paranaense, 7: 1-216.

Morretes, F.L. 1952. Novas espécies brasileiras da família Strophocheilidae. Arquivos de Zoologia do Estado de São Paulo, 8(4): 109-126.

Morretes, F.L. 1953. Addenda e corrigenda ao ensaio de catálogo dos moluscos do Brasil. Arquivo do Museu Paranaense, 10: 37-76. 
Nascimento, D.B. \& Foleto, E.M. 2011. Incompatibilidade legal em áreas protegidas na cidade de Santa Maria, RS, Brasil. Revista Bibliográfica de Geografía y Ciencias Sociales, 16: 943.

Olazarri, J.; Mones, A. \& Duarte, E. 1972. Lista de los ejemplares-tipo depositados en el Museo Nacional de Historia Natural de Montevideo, Uruguay. II. Mollusca. Comunicaciones Zoologicas del Museo de Historia Natural de Montevideo, 133(10): 1-8.

Oliveira, M.P. \& Almeida, M.N. 1999. Conchas dos caramujos terrestres do Brasil. Juiz de Fora, Editar Editora Associada. 61 p.

Oliveira, M.P. \& Oliveira, M.H.R. 1984. Comunicações Malacológicas no. 16: Listagem de Tipos de gastropoda Pulmonata brasileiros depositados em quatro Museus Europeus. Boletim do Instituto de Ciências Biológicas e Geociências, 38: 1-46.

Oliveira, M.P.; Silveira e Sá, R.C. \& Bessa, E.C.A. 1992. Catálogo dos moluscos da Universidade Federal de Juiz de Fora. Juiz de Fora, Editora da UFJF. $156 \mathrm{p}$.

Oliveira, M.P.; Vieira, I.; Oliveira, M.H.R. \& Rodrigues, G.J. 1972. Comunicações malacológicas № 7. Contribuição ao conhecimento das conchas brasileiras, litoral do Espirito Santo. Boletim do Instituto de Ciências Biológicas e de Geociências, 4: 1-18.

Parodiz, J.J. 1946a. Contibuciones al conocimiento de los moluscos terrestres sudamericanos, IV. Comunicaciones Zoologicas del Museo de Historia Natural de Montevideo, 2(27): 1-13.

Parodiz, J.J. 1946b. Los gêneros de los Bulimulidae Argentinos. Revista del Museo de la Plata (nueva serie), 4: 303-371.

Parodiz, J.J. 1957a. Catalogue of the land Mollusca of Argentina. Nautilus, 70(4): 127-135.

Parodiz, J.J. 1957b. Catalogue of the land Mollusca of Argentina. Nautilus, 71(1): 22-30.

Parodiz, J.J. 1962. New and Little-known Species of South and Central American Land Snails (Bulimulidae). Proceedings of the United States National Museum, 113(3462): 429-456.

Pfeiffer, L. 1841. Symbolae ad historiam Heliceorum. Cassellis, Sumptibus \& Typis Th. Fisheri. $88 \mathrm{p}$.

Pfeiffer, L. 1842. Symbolae ad historiam Heliceorum, Sectio Altera. Cassellis, Sumptibus \& Typis Th. Fisheri. $147 \mathrm{p}$.

Pfeiffer, L. 1846. Die Schnirkelschiiecken nebst den zunächst verwandten gattungen. In: Martini \& Chemnitz. Sytematisches Conchylien Cabinet. Nürnberg. v. 12, pt. 3, p. 285-524.

Pfeiffer, L. 1848. Monographia Heliceorum Viventium. Lipsiae, F.A. Brockhaus. v. 2, 594 p.

Pfeiffer, L. 1853. Monographia Heliceorum Viventium. Lipsiae, F.A. Brockhaus. v. $3,711 \mathrm{p}$.

Pfeiffer, L. 1854. Descriptions of sixty-six new land shells, from the collection of Hugh Cuming Esq. Proceedings of the Zoological Society of London, 1852: 56-70.

Pfeiffer, L. 1855. Versuch einer Anordnung der Heliceen nach natürlichen Gruppen. Malakozoolosgische Blätter, 2: 112-188.

Pfeiffer, L. 1876. Monographia heliceorum viventium. Lipsiae, F.A. Brockhaus. v. 7, $674 \mathrm{p}$

Picoral, M. \& Thomé, J.W. 1989. Sobre a anatomia do sistema genital de Bradybaena similaris (Férussac, 1821) (Pulmonata, Stylommatophora, Bradybaenidae) ocorrentes em Porto Alegre, estado do Rio Grande do Sul, Brasil. Memórias do Instituto Oswaldo Cruz, 84(4): 435-439.

Pilsbry, H.A. 1897-1898. American Bulimulidae: Bulimulus, Neopetraeus, Oxychona, and South American Drymaeus. Manual of conchology, Serie 2. Philadelphia, GWTJ, v. 11, 399 p.

Pilsbry, H.A. 1899. American Bulimulidae: North Ameriacan and Antillean, Drymaeus, Leiostracus, Orthalicinae and Amphibuliminae. Manual of conchology, Serie 2. Philadelphia, GWTJ, v. 12, 258 p.
Pilsbry, H.A. 1900. New South American land shells. Proceedings of the Academy of Natural Sciences of Philadelphia, 52: 385-394.

Pilsbry, H.A. 1901-1902. Pulmonata. In: Tryon, G.W. Manual of conchology (second series). Philadelphia, GWTJ. v. 14, 302 p. +62 pls.

Pilsbry, H.A. 1906. Achatinidae: Stenogyrinae and Coeliaxinae. In: Tryon, G.W. Manual of Conchology (second series), Pulmonata). Philadelphia, GWTJ. v. $18.357 \mathrm{p}$.

Pilsbry, H.A. 1911. Non-marine Mollusca of Patagonia. In: Scott, W.B. (Ed.). Reports of the Princenton University Expeditions to Patagonia 1896-1899. Princeton, Princeton University. p. 513-633 + pls. 38-47.

Pilsbry, H.A. 1920-1921. Pupillidae: Vertigininae and Pupillinae. In: Tryon, G.W. Manual of Conchology (second series): Pulmonata. Philadelphia, GWTJ. v. 26: 254 p. +24 pl.

Pilsbry, H.A. 1930a. Results of the Pinchot south sea expedition - II. Land mollusks of the canal zone The Republic of Panama, and the Cayman Islands. Proceedings of the Academy of Natural Sciences of Philadelphia, 82: 339-354.

Pilsbry, H.A. 1930b. South American land and freshwater mollusks: notes and description. VII. Proceedings of the Academy of Natural Sciences of Philadelphia, 82: 355-365.

Pilsbry, H.A. 1946. Land Mollusca of North America (North of México). Academy of Natural Sciences of Philadelphia Monographs, 2(1): 1-520.

Pitoni, V.L.L.; Veitenheimer, I.L. \& Mansur, M.C.D. 1976. Moluscos do Rio Gramde do Sul: coleta, preparação e conservação. Iheringia Serie Divulgação, 5: 25-68.

Potiez, V.L. \& Michaud, A.L.G. 1838. Galérie des Mollusques, ou catalogue méthodique, descriptif et raisonné des mollusques et coquilles du Muséum de Douai. J.B. Bailliére, Paris 1:560 p.

Quintana, M.G. 1982. Catálogo preliminar de la malacofauna del Paraguay. Revista del Museo Argentino de Ciencias Naturales "Bernardino Rivadavia" (Zoologia), 11(3): 61-158.

Ramírez, R.; Paredes, C. \& Arenas, J. 2003. Moluscos del Perú. Revista de Biologia Tropical, 51(3): 225-284.

Reeve, L.A. 1849a. Monograph of the genus Achatina. In: Conchologia Iconica, or illustrations of the shells of molluscous animals. London, Reeve. v. 5, 23 pls.

Reeve, L.A. 1849b. Monograph of the genus Bulimus. In: Conchologia Iconica, or illustrations of the shells of molluscous animals. London, Reeve. v. 5 , $89 \mathrm{pls}$.

Reeve, L.A. 1854. Monograph of the genus Helix. In: Conchologia Iconica, or illustrations of the shells of molluscous animals. London, Reeve. v. 7 , $210 \mathrm{pls}$.

Reeve, L.A. 1862. Monograph of the genus Simpulopsis. In: Conchologia Iconica, or illustrations of the shells of molluscous animals. London, Reeve. v. 13: 2 pls.

Richards, H.G. \& Hummelinck, P.W. 1940. Land and freshwater mollusks from Magarita Island, Venezuela. Notula Naturae, 62: 1-16.

Richardson, C.L. 1995. Bulimulidae: catalog of species. Tryonia, 28: i-iii, 1-458.

Rumi, A.; Sánchez, J. \& Ferrando, N.S. 2010. Theba pisana (Müller, 1774) (Gastropoda, Helicidae) and other alien land molluscs species in Argentina. Biological Invasions, 12: 2985-2990.

Salgado, N.C. \& Coelho, A.C.S. 2003. Moluscos terrestres do Brasil (gastrópodos operculados ou não, exclusive Veronicellidae, Milacidae e Limacidae). Revista de Biología Tropical, 51(3): 149-189.

Salvador, R.B. 2019a. Brazilian, Uruguayan and Argentinian land snails in the collection of the Museum of New Zealand Te Papa Tongarewa. Tuhinga, 30: 82-98.

Salvador, R.B. 2019b. Land snail diversity in Brazil. Strombus, 25: 10-20.

Salvador, R.B.; Cavallari, D.C. \& Simone, L.R.L. 2015. Taxonomical study on a sample of land snails from southeastern Tocantins state, Brazil, with description of a new species. Journal of Conchology, 42: 67-78. 
Salvador, R.B.; Cavallari, D.C. \& Simone, L.R.L. 2016. Taxonomical study on a sample of land snails from Alto Ribeira State Park (São Paulo, Brazil), with description of a new species. Archiv für Molluskenkunde, 145(1): 59-68.

Salvador, R.B.; Cavallari, D.C. \& Simone, L.R.L. 2017. Taxonomical study on a sample of land and freshwater snails from caves in central Brazil, with description of a new species. Zoosystematics and Evolution, 93(1): 135-141.

Salvador, R.B.; Charles, L.; Simone, L.R.L. \& Maestrati, P. 2018a. Terrestrial gastropods from Pedra Talhada Biological Reserve, Alagoas state, Brazil, with description of a new species of Radiodiscus (Gastropoda: (haropidae). Archiv für Molluskenkunde, 147(1): 101-128.

Salvador, R.B.; Colley, E. \& Simone, L.R.L. 2018b. Terrestrial mollusks from the region of Corumbá and Maciço do Urucum, SW Brazil. Journal of Conchology, 43(1): 71-88.

Salvador, R.B.; Silva, N.G.; Alves, R.J.V.; Moura, R.L. \& Simone, L.R.L. 2014. New records of Helicina inaequistriata (Gastropoda: Helicinidae) from Rio de Janeiro and São Paulo states, Brazil. Check List, 10: 936-938.

Santos, 0. 1994. Presencia de Bradybaena similaris (Férussac, 1821) en el Uruguay. Comunicaciones de la Sociedad Malacologica del Uruguay, 7(66-67): 376-378.

Santos, S.B.; Rodrigues, C.L.; Nunes, G.K.M.; Miyahira, I.C.;Viana, T.A.; Oliveira, J.L.; Fonseca, F.C. \& Silva, P.S.C. 2010. Estado do conhecimento da fauna de invertebrados não-marinhos da llha Grande (Angra dos Reis, RJ). Oecologia Australis, 14(2): 504-549.

Sawaya, P. \& Petersen, J.A. 1962. Sobre a ocorrência de Strophocheilidae (molusco gastrópode) no Rio Grande do Sul. Boletim da Faculdade de Ciências e Letras da USP (Zoologia), 24(261): 31-42.

Scarabino, F. 2003. Lista sistemática de los gastropoda terrestres vivientes de Uruguay. Comunicaciones de la Sociedad Malacológica del Uruguay, 8(78-79): 203-214.

Scarabino, F. 2004. Conservación de la malacofauna uruguaya. Comunicaciones de la Sociedad Malacológica del Uruguay, 8(82-83): 267-273.

Scopoli, G.A. 1786. Delicia e florae et fauna insubricae sev novae, aut minus cognitae species plant arumet animalium in insubria austríaca. Ticini. Pt. 1,85 p.

Shade, F.H. 1965. Lista de moluscos del Guaira (Villarrica-Paraguay) conocidos hasta el presente. Comunicaciones de la Sociedad Malacológica del Uruguay, 1(8): 209-221.

Silva, C.C. \& Castro, G.A. 2003. Gastrópodes terrestres num fragmento de restinga no estado do Espírito Santo. Bioikos, 17(1/2): 65-69.
Simone, L.R.L. 1999. Mollusca Terrestres. In: Brandão, C.R.; Cancello, E.M. (Eds.). Biodiversidade do Estado de São Paulo, Brasil: síntese do conhecimento ao final do século XX. São Paulo, FAPESP. v. 5: 3-8.

Simone, L.R.L. 2006. Land and freshwater mollusks of Brazil. São Paulo, EGB/ FAPESP. $390 \mathrm{p}$.

Smith, E.A. 1903. Note on some mollusks of the Family Bulimulidae from Mato Grosso (Percy Sladen Expedition to Central Brazil). Proceedings of the Zoological Society of London, 2: 70-71.

Sowerby, G.B. 1824. Descriptions, accompanied by figures of several new epscies of shells. The Zoological Journal of Linnean Society, 1: 56-60.

Spix, J.B. 1827. Testacea fluviatilia quae in itinere per Brasiliam annis MDCCCXVII-MDCCCXX jussu et auspiciis Maximiliani Josephi I. Bavariae regis augustissimi suscepto collegit et pingenda curavit Dr. J.B. de Spix, digessit, descripsit et observationibus illustravit Dr. J.A. Wagner. Munich, C. Wolf. $36 \mathrm{p}$.

Strobel, P. 1874. Materiali per una malacostatica di terra e di acqua dolce dell'Argentinia Meridionale. Biblioteca Malacologica, 4: I-LXXX + 142 p.

Suter, H. 1900: Observações sobre alguns caracóis terrestres do Brasil. Revista do Museu Paulista, 4:339-337.

Swainson, W. 1840. A treatise on Malacology or the natural classification of shells and shellfish. London. $419 \mathrm{p}$.

Thiele, J. 1927: Über einige brasilianische Landschnecken. Abhandlungen der Senckenbergischen Naturforschenden Gesellschaft, 40(3): 307-330.

Thompson, F.G. 2011. An annotated checklist and bibliography of the land and freshwater snails of México and Central America. Bulletin of the Florida Museum of Natural History, 50(1): 1-299.

Tillier, S. 1980. Gastéropodes terrestres et fluviatiles de Guyane Française. Mémoires du Muséum national d'Histoire naturelle A - Zoologie, 118: 1-189.

Troschel, F.H. 1849. Ueber die mundthiele einiger heliceen. Archiv für Naturgeschichte, 15: 225-236.

Vernhout, J.H. 1914. The non-marine mollusks of Surinarn. Notes from the Leyden Museum, 36(1): 1-46.

Wagner, A.J. 1910. Die Familie Helicinidae, N.F. In: Martini F.H. \& Chemnitz, J.W., 1837-1920. Systematische Conchilien Cabinet. Nürberg, Bauer \& Raspe. v. 1, pt. 18, p. 265-368.

Weyrauch, W.K. 1966. Gastropodos terrestres de Argentina, Uruguay y Brasil. Neotropica, 12(38): 41-47.

Zilch, A. 1960. Euthyneura. In: Schindewolf, 0.H. (Ed.). Handbuch der Paläozoologie. Berlin, Borntraeger. v. 6, pt. 2, p. 401-600. 